\title{
Band-Gap Deformation Potential and Elasticity Limit of Semiconductor Free-Standing Nanorods Characterized in Situ by Scanning Electron Microscope-Cathodoluminescence Nanospectroscopy
}

\author{
Kentaro Watanabe, ${ }^{*, t, \neq}$ Takahiro Nagata, ${ }^{\dagger}$ Yutaka Wakayama, ${ }^{\dagger}$ Takashi Sekiguchi, ${ }^{\dagger}$ Róbert Erdélyi, ${ }^{\S}$ and \\ János Volk ${ }^{\S}$ \\ ${ }^{\dagger}$ WPI Center for Materials Nanoarchitectonics, National Institute for Materials Science, 1-1 Namiki, Tsukuba, Ibaraki 305-0044, Japan, ${ }^{\ddagger}$ Faculty of Pure and \\ Applied Sciences, University of Tsukuba, 1-1-1 Tennodai, Tsukuba, Ibaraki 305-8571, Japan, and ${ }^{\S}$ MTA EK Institute of Technical Physics and Materials Science, \\ Konkoly Thege M. út 29-33, 1121 Budapest, Hungary
}

\begin{abstract}
Modern field-effect transistors or laser diodes take advantages of band-edge structures engineered by large uniaxial strain $\varepsilon_{z z}$ available up to an elasticity limit at a rate of band-gap deformation potential $a_{z z}\left(=\mathrm{d} E_{\mathrm{g}} / \mathrm{d} \varepsilon_{z z}\right)$. However, contrary to $a_{\mathrm{p}}$ values under hydrostatic pressure, there is no quantitative consensus on $a_{z z}$ values under uniaxial tensile, compressive, and bending stress. This makes band-edge engineering inefficient. Here we propose SEM-cathodoluminescence nanospectroscopy under in situ nanomanipulation

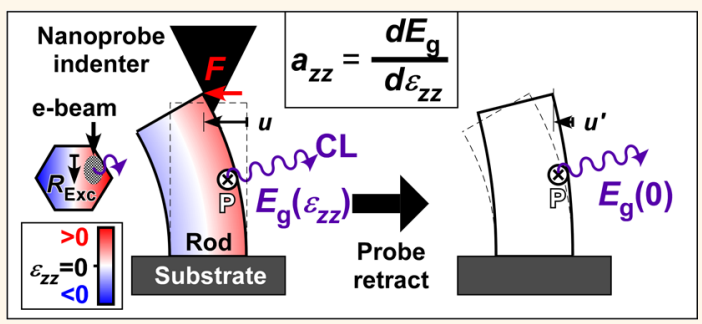
(Nanoprobe-CL). An apex of a c-axis-oriented free-standing $\mathrm{Zn} 0$ nanorod (NR) is deflected by point-loading of bending stress, where local uniaxial strain $\left(\varepsilon_{c c}=r / R\right)$ and its gradient across a NR $\left(\mathrm{d} \varepsilon_{c c} d \mathrm{~d} r=R^{-1}\right)$ are controlled by a NR local curvature $\left(R^{-1}\right)$. The NR elasticity limit is evaluated sequentially $\left(\varepsilon_{c c}=0.04\right)$ from SEM observation of a NR bending deformation cycle. An electron beam is focused on several spots crossing a bent NR, and at each spot the local $E_{\mathrm{g}}$ is evaluated from near-band-edge $C L$ emission energy. Uniaxial $a_{c c}\left(=\mathrm{d} E_{\mathrm{g}} / \mathrm{d} \varepsilon_{c c}\right)$ is evaluated at regulated surface depth, and the impact of $R^{-1}$ on observed $a_{c c}$ is investigated. The $a_{c c}$ converges with $-1.7 \mathrm{eV}$ to the $R^{-1}=0$ limit, whereas it quenches with increasing $R^{-1}$, which is attributed to free-exciton drift under transversal band-gap gradient. Surface-sensitive $\mathrm{CL}$ measurements suggest that a discrepancy from bulk $a_{c c}=-4 \mathrm{eV}$ may originate from strain relaxation at the side surface under uniaxial stress. The nanoprobe-CL technique reveals an $E_{\mathrm{g}}\left(\varepsilon_{i j}\right)$ response to specific strain tensor $\varepsilon_{i j}(i, j=x, y, z)$ and strain-gradient effects on a minority carrier population, enabling simulations and strain-dependent measurements of nanodevices with various structures.
\end{abstract}

KEYWORDS: in-situ scanning electron microscopy $\cdot$ cathodoluminescence $\cdot$ plastic deformation $\cdot$ deformation potential $\cdot \mathrm{ZnO} \cdot$ free-standing nanowire $\cdot$ surface elasticity

\begin{abstract}
C train engineering of semiconductor
$\checkmark$ pro band-edge structure is a major ap-

proach to advance nanodevices, such as field-effect transistors (FETs) with enhanced carrier mobility, ${ }^{1,2}$ laser diodes (LDs) with reduced lasing threshold and gain, ${ }^{3}$ wavelength tuning of optoelectronic devices including solar cells, ${ }^{4,5}$ and nanopiezotronics of piezoelectric materials. ${ }^{6}$ Therein, band-edge parameters, such as band gap, $E_{g}\left(\varepsilon_{i j}\right)(i, j=x, y, z)$, and carrier effective mass, $m_{k l}\left(\varepsilon_{i j}\right)(i, j, k, I=x, y, z)$, couple
\end{abstract}

material mechanics and band-edge structures, which are tuned by strain, $\varepsilon_{i j}$ (secondrank strain tensor), applicable up to fracture strain, $\varepsilon_{i j \mathrm{~F}}$ (or a yield strain $\varepsilon_{i j \gamma}$ ). Technical progress of crystal growth and large strain retention realize single-crystalline semiconductor nanowires (NWs) retaining uniaxial strain $\left(\varepsilon_{z z}\right)$ up to $\varepsilon_{z z F} \approx 10^{-1}$, larger than bulk $\left(\varepsilon_{\mathrm{zzF}} \approx 10^{-3}\right)$ or a lattice-mismatched heteroepitaxial thin film $\left(\varepsilon_{z z F} \approx 10^{-2}\right)$. Thus, NW-based devices make strain engineering even more significant. *Address correspondence to
Watanabe.Kentaro@nims.go.jp.

Received for review December 16, 2014 and accepted February 17, 2015.

Published online

10.1021/nn507159u

(c) XXXX American Chemical Society 
However, contrary to hydrostatic pressure, anisotropic stress (e.g., uniaxial stress and shear stress) impact on strain distributions $\varepsilon_{i j}(x, y, z)$ in a structure is less evident for crystals in nanoscale or at surfaces. More significantly, band-edge parameter responses to anisotropic strain are unsettled (e.g., band-gap deformation potentials $\left(a_{i j} \equiv \mathrm{d} E_{\mathrm{g}} / \mathrm{d} \varepsilon_{i j}\right.$ at $\left.\varepsilon_{i j}=0\right)$ for uniaxial $\varepsilon_{z z}$ $\left(a_{z z}\right)$ and that for shear $\left.\varepsilon_{x z}\left(a_{x z}\right)\right)$. This results in difficulties of band-edge strain engineering to realize devices with intended properties. Microscopic strain distribution in a device structure, $\varepsilon_{i j}(x, y, z)$, is measurable nowadays by high-resolution TEM, $\mu$-Raman spectroscopy, etc. Expected device properties may be simulated numerically for their efficient development, starting from strain distribution $\varepsilon_{i j}(x, y, z)$ present in the structure. However, unsettled parameters disable such attempts.

For instance, wurzite $\mathrm{ZnO}$ is a suitable semiconductor for the above applications, which has a direct band gap $\left(E_{\mathrm{g}}=3.37 \mathrm{eV}\right)$, a large exciton binding energy $(60 \mathrm{meV}){ }^{7}$ and a large piezoelectric constant along the polar $\langle 0001\rangle c$-axis $\left(d_{c c}=12.4 \mathrm{pm} / \mathrm{V}\right){ }^{8} \mathrm{ZnO}$ under hydrostatic pressure is well understood below its transient pressure $(P=10 \mathrm{GPa})$ to a rock-salt structure. ${ }^{9}$ Photoabsorption (PA) ${ }^{10,11}$ or photoluminescence $(\mathrm{PL})^{12,13}$ spectroscopy in a diamond anvil cell revealed that hydrostatic $a_{\mathrm{p}}\left[\equiv \mathrm{d} E_{\mathrm{g}} / \mathrm{d}(\ln V)\right]$ converges between -3.5 and $-4.5 \mathrm{eV}$, regardless of crystal volume $V$ and dimensions (nanowire, thin film, and bulk). In contrast, $\mathrm{ZnO}$ under c-axial stress is less understood. Numerical simulations predicted structure transitions to a graphitelike structure ${ }^{14,15}$ at $\varepsilon_{c c}<-0.15$ and to a layered structure $^{14,16}$ or to a body-centered tetragonal structure ${ }^{17}$ at $\varepsilon_{c c}>0.06$. Mechanical properties of wurzite $\mathrm{ZnO} \mathrm{NWs}^{17-24}$ or nanobelts (NBs) ${ }^{25,26}$ are investigated under uniaxial tensile stress, ${ }^{17-20}$ compressive and shear stress, ${ }^{21}$ bending stress, ${ }^{20,23-25}$ or dynamic mechanical resonance. ${ }^{22,26}$ Also, high-resolution TEM observation of a strain-free $\mathrm{ZnO} N W$ surface revealed $\mathrm{ZnO}(10-10) \mathrm{m}$ surface reconstruction with a radial contraction up to $\varepsilon_{\text {aa }}=-0.061$ at the surface to a depth of $t_{\text {Surf }}=1.3 \mathrm{~nm}^{27}$ Some authors ${ }^{18,20-22}$ report significant increases of $c$-axial NW Young's moduli at diameters $D<10^{2} \mathrm{~nm}$, and they attributed it to $\mathrm{ZnO}$ surface reconstruction, while other authors ${ }^{24-26}$ report no significant $D$-dependences. Uniaxial $a_{c c}\left(\equiv \mathrm{d} E_{\mathrm{g}} /\right.$ $\left.\mathrm{d} \varepsilon_{c c}\right)$ of $\mathrm{ZnO}$ bulk, $^{28-32}$ microwire $(\mathrm{MW}),^{33-36,38}$ or $\mathrm{NW}^{29,33,37,42,43}$ under uniaxial tensile/compressive stress $^{28-32}$ or uniaxial bending stress ${ }^{35-43}$ is studied by numerical calculations $s^{28,29}$ or near-band-edge (NBE) spectroscopy of photoreflectance (PR), ${ }^{30,31} \mathrm{PA}^{32}$ $\mu$ - $\mathrm{PL},{ }^{35,36}$ or cathodoluminescence $(\mathrm{CL})$ at high electron beam energy (e-beam, $E_{\mathrm{PE}} \approx 10 \mathrm{keV}$ ). ${ }^{37-43}$ Unlike hydrostatic $a_{\mathrm{p}}$, there is no quantitative consensus on uniaxial $a_{c c}$ values reported in a wide range between -3.96 and $-0.37 \mathrm{eV}$ (Figures 4 and S3). Thus, we need a novel technique that characterizes uniaxial $a_{c c}$ explicitly (Supplementary Note 1) and examines underlying factors: stress loading mode dependence and surface elasticity impacts.

This study focuses on the band-gap response to applied uniaxial strain at a controlled uniaxial strain gradient. We have developed a SEM-CL nanospectroscopy in combination with in situ nanomanipulation using a W-nanoprobe indenter (Nanoprobe-CL). Welldefined uniaxial strain and strain gradient are applied to $c$-axis-oriented free-standing $\mathrm{ZnO}$ nanorods (NRs), and they are controlled up to large values by loading point bending stress at the NR apex. Local $E_{\mathrm{g}}$ is evaluated explicitly by high-resolution $C L$ spectroscopy, where in-plane resolution and surface depth $\left(R_{\mathrm{Exc}}\right)$ of the $C L$ probe are regulated by primary e-beam energy of SEM. NR bending deformation is observed by SEM, and nominal $\varepsilon_{c \mathrm{NN}}$ and its spatial gradient $\mathrm{d} \varepsilon_{c \mathrm{NN}} /$ $\mathrm{d} r\left(=R^{-1}\right.$ : NR local curvature) are calculated using a continuum mechanical formulation. Plastic $\varepsilon_{c c \mathrm{P}}$ is also evaluated by observing residual NR deformation after unloading the stress. Elastic $\varepsilon_{c C E}\left(=\varepsilon_{c c N}-\varepsilon_{c C P}\right)$ values with respect to nominal $\varepsilon_{c c N}$ in a bent NR are plotted, and the NR elasticity limit is evaluated from statistical $\left(\varepsilon_{c C E}, \varepsilon_{c c N}\right)$ plots, to ensure NR elastic deformation at each bending deformation. Local $E_{\mathrm{g}}$ due to $\varepsilon_{c c \mathrm{~N}}$ is evaluated by near-band-edge $C L$ spectroscopy at each spot on a bent NR with a local $R^{-1}$, from which $a_{c c \mathrm{~N}}\left[\equiv \mathrm{d} E_{\mathrm{g}} / \mathrm{d} \varepsilon_{c c \mathrm{~N}}\right]$ at each $R^{-1}$ is evaluated.

\section{RESULTS AND DISCUSSION}

NRs under Bending Deformation Cycles and $\delta E_{g}$ Response to Nominal $\varepsilon_{c \mathrm{cN}}$ at Different Local $\boldsymbol{R}^{-\mathbf{1}}$. Free-standing $\mathrm{ZnO}$ NRs of uniform diameters $(D=0.15 \mu \mathrm{m})$ and lengths $(L=1.4 \mu \mathrm{m})$ under bending deformation cycles are observed by SEM, where room-temperature $\left(\varepsilon_{c c \mathrm{~N}}, \delta E_{\mathrm{g}}\right)$ plots are evaluated at each NR curvature $R^{-1}$ (Figures 1, 2, and S1). At each moment in the NR bending and releasing cycle, NR lateral deflection $u$ is evaluated from a SEM image. The outer bottom of a bent NR, position $\mathrm{P}_{0}$, has the largest tensile strain. For a concise description, NR bending magnitude is represented by nominal uniaxial strain at $\mathrm{P}_{0}: \varepsilon_{c c N, 0}=\left(3 D / 2 L^{2}\right) u$. Plastic uniaxial strain at $\mathrm{P}_{0}$ is also evaluated: $\varepsilon_{c c \mathrm{P}, 0}=\left(3 D / 2 L^{2}\right) u^{\prime}$. Simultaneously, spot-CL spectroscopy "runs" across a bent section of a NR with a local curvature $R^{-1}$. In each "run", the e-beam is spotted sequentially at each position $\mathrm{P}_{k}(k=1,2, \ldots, N)$, at which the local $\mathrm{CL}$ spectrum is recorded. Strain-induced band-gap energy shift at $P_{k}$ is evaluated from an energy shift of a freeexciton ( $\mathrm{FX}) \mathrm{CL}$ peak due to $\mathrm{NR}$ bending: $\delta E_{\mathrm{g}}=$ $E_{\mathrm{FX}}-E_{\mathrm{FX}}{ }^{0}$. Obtained $N$ plots of $\left(\varepsilon_{\mathrm{ccN} N}, \delta E_{\mathrm{g}}\right)$ are line-fitted to evaluate a $C L$ peak shift excited at the NR center $(r=0), \delta E_{g}(0)$, and the nominal band-gap deformation potential, $a_{c c \mathrm{~N}}$, at a certain strain gradient: $\mathrm{d} \varepsilon_{c c \mathrm{~N}} /$ $\mathrm{d} r=R^{-1}$.

First, a NR under a small bending cycle up to $\varepsilon_{c \mathrm{cN}, 0}=$ 0.020 at $P_{0}$ is studied by spot-CL spectroscopy (runs $1-4$, all with $N=4$ ) (Figure $1 \mathrm{a}$ ). This NR exhibits a 


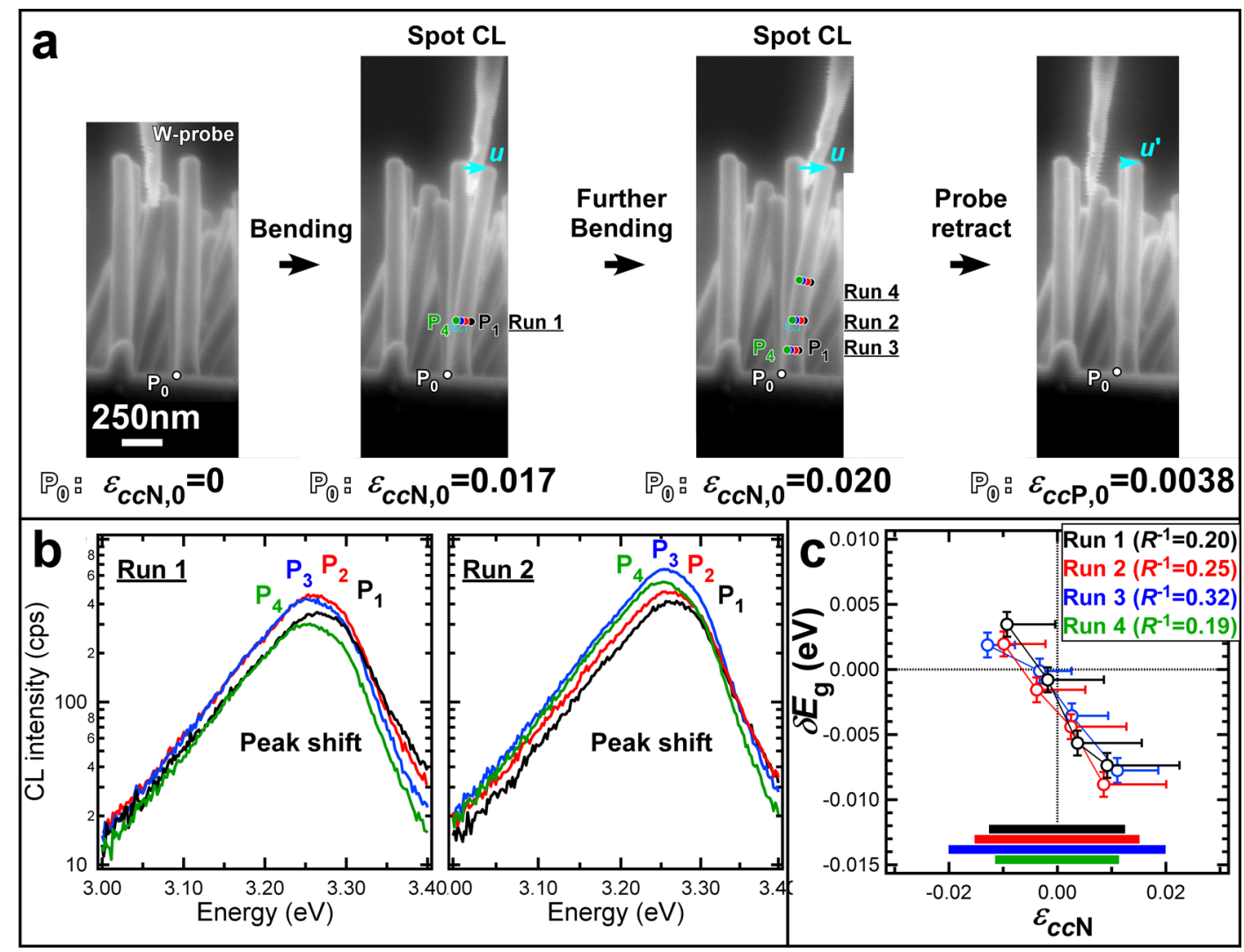

Figure 1. Spot-CL spectroscopy on a weakly bent ZnO NR. (a) Series of side-view ( $m$-axis) SEM images of a ZnO NR before bending $\left(\varepsilon_{c \mathrm{cN}, 0}=0\right.$ at $\left.\mathbf{P}_{0}\right)$, under small bending $\left(\varepsilon_{c \mathrm{cN}, 0}=0.017\right.$ at $\mathbf{P}_{0}$ in run 1 and $\varepsilon_{c \mathrm{cN}, 0}=0.020$ at $\mathbf{P}_{0}$ in runs 2,3 , and 4$)$, and after the bending $\left(\varepsilon_{c c P, 0}=0.0038\right.$ at $\left.P_{0}\right)$. Spot-CL spectroscopy is performed at each $P_{k}(k=1, \ldots, N)$ on this NR in each run $(N=4$ in any run). (b) Obtained spot-CL spectra in runs 1 and 2. (c) The $\left(\varepsilon_{c \mathrm{cN}}, \delta E_{\mathrm{g}}\right)$ plots in each run (plots from run 4 are not shown for clarity). Local NR curvature $R^{-1}$ in each run is denoted, and the $\varepsilon_{c c N}$ range available between NR outer and inner edges at height $s$ in each run $\left(\left|\varepsilon_{c c N}\right|<\varepsilon_{c c N, s}\right)$ is indicated with a corresponding color solid horizontal bar. The $\varepsilon_{c c N, 0}$ (or $\left.\varepsilon_{c c P, 0}\right)$ error, governed by SEM resolution, is \pm 0.0009 . The $\varepsilon_{c c N}$ error is governed by XYZ stage drift with respect to the e-beam during a series of spot-CL spectroscopy. The $\delta E_{\mathrm{g}}$ error, dominated by statistical $E_{\mathrm{FX}}{ }^{0}$ differences among different $\mathrm{NRs}$, is $\pm 4.1 \mathrm{meV}(n=20)$.

negligible plastic deformation $\left(\varepsilon_{c c P, 0}=0.0038\right)$. At any run, $E_{\mathrm{FX}}$ red-shifts as the position shifts from a compressive region to a tensile one (Figure $1 \mathrm{~b}$ ), and the $\delta E_{\mathrm{g}}$ red-shifts linearly with $\varepsilon_{c c N}$ (run 4 is not shown for clarity) (Figure 1c). The $a_{c \mathrm{cN}}$ is evaluated to be negative: $-0.61 \mathrm{eV}$ in run $1,-0.57 \mathrm{eV}$ in run $2,-0.39 \mathrm{eV}$ in run 3 , and $-0.41 \mathrm{eV}$ in run 4 . Similarly, the NR under the cycle of larger bending (up to $\varepsilon_{c \mathrm{cN}, 0}=0.040$ ) is studied by spot-CL spectroscopy (run 1 with $N=5$ at $\varepsilon_{c \mathrm{cN}, 0}=$ 0.0081 and run 2 with $N=4$ at $\varepsilon_{c \mathrm{CN}, 0}=0.038$ ) (Figure 2a). This NR finally fractured at a c-plane cleavage indicated by the green arrow $\left(\varepsilon_{c c F, 0}=0.04\right)$. This is due to the stress concentration at the position spatially distant from both NR apex (loading point) and bottom (clamping plane), demonstrating the utility of our setup for NR fracture tests. Spot-CL spectroscopy is performed on NRs under small and large bending. The linear $\delta E_{\mathrm{g}}$ red shifts with $\varepsilon_{c \mathrm{cN}}$ are observed even at small bending (run 1); however, it is no longer observable at larger bending (run 2) (Figure $2 \mathrm{~b}$ and $\mathrm{c}$ ). The $a_{c \mathrm{NN}}$ is evaluated to be $-0.98 \mathrm{eV}$ in run 1 and $0.058 \mathrm{eV}$ in run 2 , where the latter seems to be quenched. Some NRs under the cycle of large bending (up to $\varepsilon_{C C N, O}=0.061$ ) and releasing exhibit a large bending deformation $\left(\varepsilon_{c c P, 0}=0.032\right)$ (Figure S1a). This is due to the stress concentration at the region indicated by a green arrow, which is highlighted by two crossing straight axes (dashed black lines) of the bent NR. Spot CL-spectroscopy is performed on NRs under bending (runs 1 and 2, each with $N=5$ at $\varepsilon_{c \mathrm{CN}, 0}=0.061$ ). Also in this case, the $\delta E_{\mathrm{g}}$ red shift with $\varepsilon_{c \mathrm{cN}}$ is not clearly observed and the $a_{c c N}$ is negligible: $0.044 \mathrm{eV}$ in run 1 and $0.036 \mathrm{eV}$ in run 2 (Figure S1b and c). As demonstrated above, NR bending deformation is elastic and the local $\mathrm{ZnO}$ band-gap energy red-shifts with a $c$-axial strain at small $\varepsilon_{c \mathrm{CN}, 0}$. However, as $\varepsilon_{c \mathrm{cN}, 0}$ increases, the NR fractures or yields (plastic deformation) and the observed $a_{c \mathrm{cN}}$ seems to be quenched.

Statistical Studies: NR Plastic Deformation with NR Bending. An accurate $a_{c c}$ evaluation is based on an explicit evaluation of NR fracture or yield strain. Thus, statistical $\left(\varepsilon_{C C N, O}, \varepsilon_{C C E, O}\right)$ plots are obtained from several bending tests of NRs (Figure $3 a$ ). The $\varepsilon_{c c P, 0}$ at each NR bending is indicated as a vertical deviation of the plot from the elastic deformation line (dashed black line): $\varepsilon_{c c E, 0}=$ $\varepsilon_{c \mathrm{CN}, 0}$. At $\varepsilon_{c \mathrm{NN}, 0}<0.02$, the $\varepsilon_{c \mathrm{EE}, 0}$ is within its error bars 


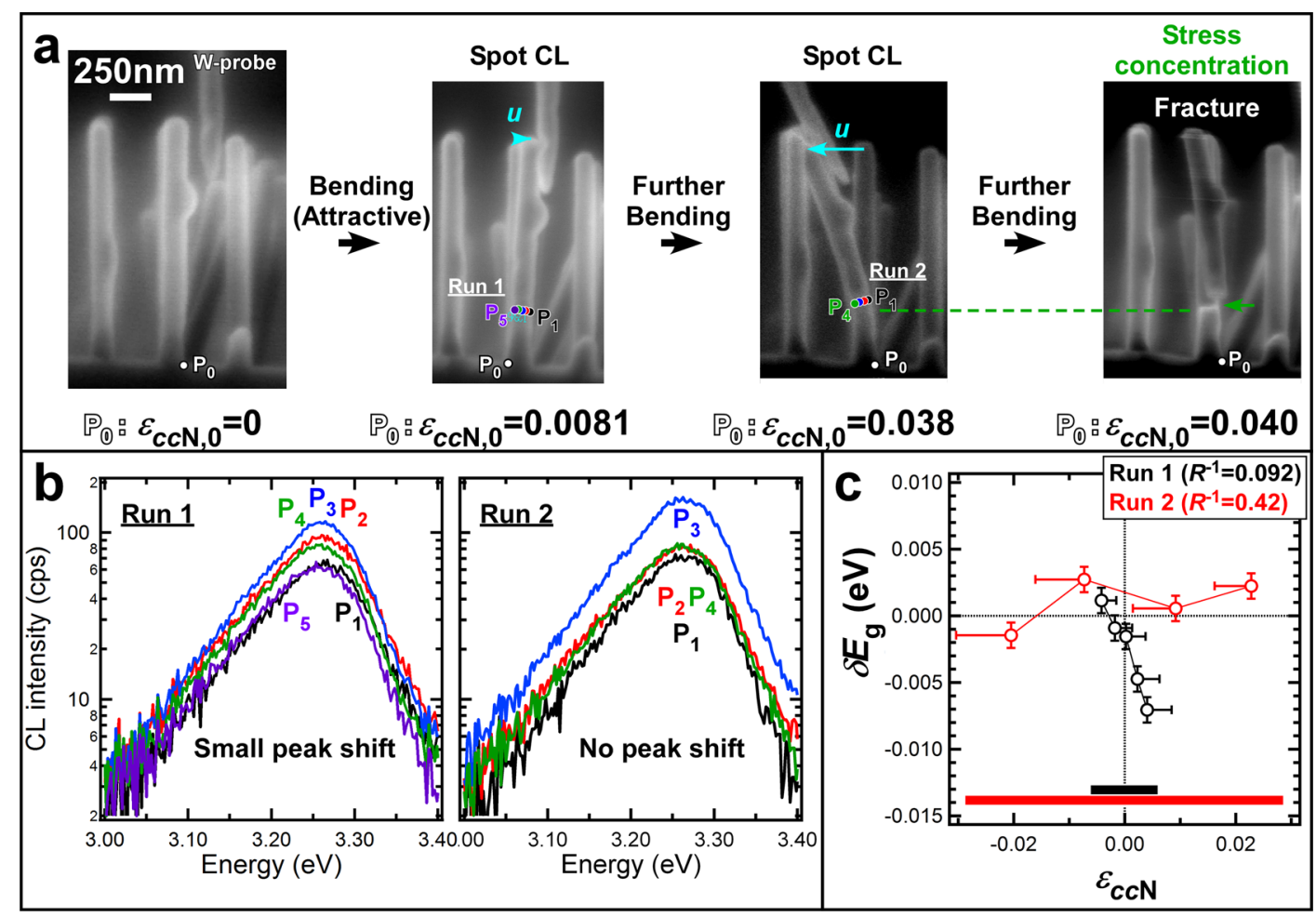

Figure 2. Spot-CL spectroscopy on a largely bent ZnO NR followed by its fracture. (a) Series of side-view ( $m$-axis) SEM images of a $\mathrm{ZnO} N R$ before bending $\left(\varepsilon_{\mathrm{ccN}, 0}=0\right.$ at $\left.\mathrm{P}_{0}\right)$, under small bending $\left(\varepsilon_{\mathrm{ccN}, 0}=0.0081\right.$ at $\mathrm{P}_{0}$ in run 1$)$, under large bending $\left(\varepsilon_{\mathrm{ccN}, 0}=\right.$ 0.038 at $P_{0}$ in run 2), and after NR fracture $\left(\varepsilon_{c c N, 0}=0.040\right.$ at $P_{0}$ just before the fracture). The fracture planes are $c$-planes and are located close to the NR bottom. Spot-CL spectroscopy is performed on this NR under small ( $N=5$ in run 1$)$ and large $(N=4$ in run 2) bending deformation. (b) Obtained $\mathrm{CL}$ spectra in runs 1 and 2. (c) The $\left(\varepsilon_{c c N}, \delta E_{\mathrm{g}}\right)$ plots in runs 1 and 2. All panels are displayed in the same manner as Figure 1.

from the elastic line and the NR bending deformation is fully elastic. At $0.02<\varepsilon_{c \mathrm{cN}, 0}<0.04$, some NRs remain fully elastic, whereas other NRs exhibit an $\varepsilon_{c c E, 0}$ deviation from the elastic line (Supplementary Note 2). However, the elastic deformation still dominates over the plastic one in any NR. In both cases, NR bending is smooth and no stress concentration is observed. At $\varepsilon_{c \mathrm{cN}, 0}>0.04$, some NRs fracture (NR in Figure 2, unplotted). Other NRs show the maximum $\varepsilon_{c C E, 0} \approx$ 0.04 (solid blue line), and $\varepsilon_{c c E, 0}$ drops by further bending where the plastic deformation starts to dominate over the elastic one (e.g., the NR in Figure S1). Here, typical NR yield strain, $\varepsilon_{c c Y, 0}=0.038$, is obtained by averaging the $\varepsilon_{C \mathrm{CE}, 0}$ in three plots. Both NR fracture and NR yield are attributed to the stress concentration. Thus, the nanoprobe- $C L$ technique enables us to characterize plastic deformation of a free-standing NR under in situ bending deformation cycles in a wide $\varepsilon_{c \mathrm{CN}, 0}$ range. Further, the NR $\varepsilon_{c \mathrm{CE}, 0}-\varepsilon_{c \mathrm{cN}, 0}$ curve obtained by the nanoprobe- $\mathrm{CL}$ technique is comparable with a stress - strain $\left(\sigma_{c c}-\varepsilon_{c c}\right)$ curve available by force spectroscopy. We obtained typical fracture strain $\left(\varepsilon_{c c F, 0}\right.$ $=0.04)$ or yield strain $\left(\varepsilon_{c c Y, 0}=0.038\right)$ of our $\mathrm{ZnO} N R s$, which determines the expected elasticity limit $\left(\varepsilon_{c c}=\right.$ $0.04)$. The strain regimes below and above $\varepsilon_{c \mathrm{cN}, 0}=$ 0.04 are labeled as "elastic" and "plastic" regimes, respectively.

\section{Statistical Studies: Apparent $a_{c c}$ Quench with NR Bending and} FX Drift under a Band-Gap Gradient. To evaluate the $a_{\mathrm{ccN}}$ explicitly and clarify an origin of $a_{c c N}$ quench with NR bending, room-temperature spot-CL spectroscopy is simultaneously performed statistically on several NRs under different NR bending magnitudes. Impacts of local uniaxial strain gradient $\mathrm{d} \varepsilon_{c \mathrm{cN}} / \mathrm{d} r\left(=R^{-1}\right.$ : NR local curvature) on $\delta E_{\mathrm{g}}(0)$ and $a_{\mathrm{ccN}}$ are investigated by spot$\mathrm{CL}$ spectroscopy at a height around $s \approx 400 \mathrm{~nm}$ (Figure $3 \mathrm{~b}$ and $\mathrm{c}$ ). We found that $a_{c \mathrm{NN}}$ approaches $-1.7 \mathrm{eV}$ at the $R^{-1} \rightarrow 0$ limit. From eq 14 , we conclude $a_{c c}=-1.7 \mathrm{eV}$ at the limit of uniform uniaxial stress, such as uniaxial tension or compression. Also we found that the $\delta E_{g}(0)$ shifts to a negative value and the $a_{c c N}$ quenches with increasing $R^{-1}$. This is unusual since $\mathrm{ZnO}$ band-gap deformation potential is normally a constant in the range $\left|\varepsilon_{c \mathrm{NN}}\right|<2 \times 10^{-2}$. $^{34}$ The discussion in Supplementary Note 3 denies the impact of NR plastic deformation on the $a_{c \mathrm{NN}}$ quench, since the $a_{\mathrm{ccN}}$ quenches with increasing NR bending evidently in the "elastic" regime, and elastic $a_{C C E}$, calculated from eq 14 , also exhibits a similar quench. Thus, we simply discuss the origin of $a_{c c N}$ quench and negative $\delta E_{g}(0)$ shift with increasing local $R^{-1}$.

The $\mathrm{ZnO}$ band diagram across a bent $\mathrm{NR}$ has a bandgap gradient $\left(F_{\mathrm{g}}=a_{c c} / R, a_{c c}<0\right)$ toward the NR outer edge $(r=D / 2)$ due to graded strain $\varepsilon_{c c}(r)=r / R$ 


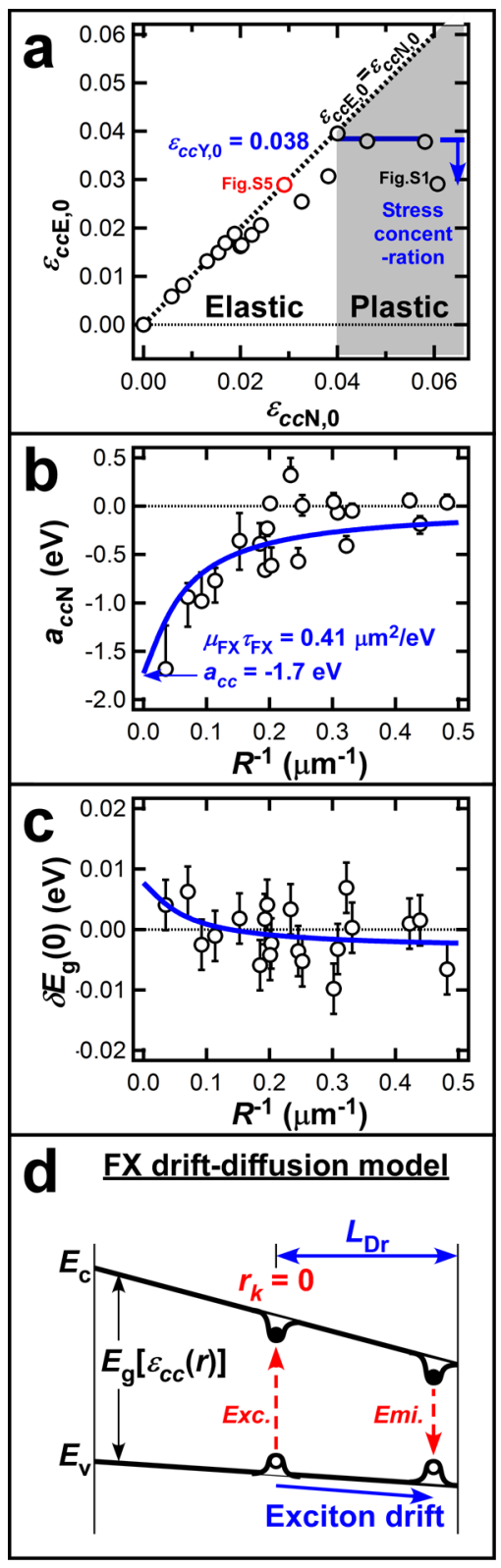

Figure 3. Statistical analyses of $\mathrm{ZnO}$ band-gap uniaxial deformation potential $a_{c c}(\mathrm{eV})$. (a) The $\left(\varepsilon_{c c N, 0}, \varepsilon_{c c E, 0}\right)$ plots evaluated at $\mathrm{P}_{0}$ (NR bottom edge), indicating the degree of NR plastic deformation at each NR bending. The plots from Figures $\mathrm{S} 1$ and $\mathrm{S} 5$ are labeled. (b) The $\left(R^{-1}, a_{\mathrm{CCN}}\right)$ plots. The FX diffusion parameter $\left(\mu_{\mathrm{Fx}} \tau_{\mathrm{FX}}=0.41 \mu \mathrm{m}^{2} / \mathrm{eV}\right)$ is evaluated by fitting the $a_{c c}\left(R^{-1}\right)$ curve (eq 2$)$ at $D=0.15 \mu \mathrm{m}$ and $a_{c c}=-1.7 \mathrm{eV}$. (c) The $\left(R^{-1}, \delta E_{\mathrm{g}}(0)\right)$ plots. They are reproduced by fitting the $\left[\delta E_{\mathrm{g}}(0)\right]\left(R^{-1}\right)$ curve (eq 3$)$ at $D=0.15 \mu \mathrm{m}$, $a_{\mathrm{cc}}=-1.7 \mathrm{eV}$, and $\mu_{\mathrm{FX}} \tau_{\mathrm{FX}}=0.41 \mu \mathrm{m}^{2} / \mathrm{eV}$. (d) FX drift-diffusion model under the band-gap gradient toward its outer edge $(r=D / 2)$. Conduction band edge $\left(E_{\mathrm{C}}\right)$ and valence band edge $\left(E_{\mathrm{V}}\right)$ are graded across a bent ZnO NR. An FX drift by distance $L_{\mathrm{Dr}}(>0)$ is illustrated. The $R^{-1}$ error, governed by SEM resolution, is $\pm 0.012 \mu \mathrm{m}^{-1}$. The $\delta E_{\mathrm{g}}(0)$ error is $\pm \mathbf{4 . 1} \mathrm{meV}$, the same as the $\delta E_{\mathrm{g}}$ error. Error bars of $a_{\mathrm{cCN}}$ (or $a_{\mathrm{cCE}}$ ) are given by the line-fitting error of the $\left(\varepsilon_{c c N}, \delta E_{\mathrm{g}}\right)$ plots and the $\varepsilon_{c \mathrm{cN}}$ (or $\varepsilon_{c \subset E}$ ) error due to $\mathrm{XYZ}$ stage drift.

(Figure 3d). Band-edge gradients may contribute to the $\delta E_{\mathrm{g}}(0)$ red shift due to the Frantz-Keldysh-like effects: carrier wave function tailing into the band gap. However, it does not alter the $a_{c c}$ at uniform strain gradient.
It is rather natural to consider minority carrier drift across a bent NR. Xu et al. observed a red-shifted NBE $\mathrm{PL}$ emission of a bent $\mathrm{ZnO} \mathrm{MW}$ under macroscopic laser excitation and accounted for it by exciton charge dissociation followed by electron drift under the transverse piezoelectric field. ${ }^{39}$ However, this picture is not reliable since radiative carrier recombination is dominated by minority carrier (hole) distribution. Our estimation suggests that an uncompensated piezoelectric field at the ZnO NR apex $\left(7.53 \times 10^{8} \mathrm{~V} / \mathrm{cm}\right)$ can be significantly larger than what is needed to dissociate $\mathrm{ZnO} \mathrm{FX}$ with a Bohr radius of $a_{\mathrm{B}}=$ $1.35 \mathrm{~nm}^{44}\left(4.4 \times 10^{5} \mathrm{~V} / \mathrm{cm}\right)$. However, such an electric field higher than the typical bulk $\mathrm{ZnO}$ breakdown field $\left(2 \times 10^{6} \mathrm{~V} / \mathrm{cm}\right)^{45}$ is not maintained, and more realistically the piezoelectric charges should be compensated by residual carriers (Supplementary Note 4). Our setup circumvents this problem and compensates the piezoelectric charge via the contacts to the grounded $\mathrm{W}$-nanoprobe and $\mathrm{ZnO}$ substrate (Figure $5 \mathrm{a}$ ). More realistically, we attribute the negative $\delta E_{g}(0)$ shift and the $a_{c \mathrm{NN}}$ quench to the transversal drift of undissociated FXs under the graded band gap.

Here we consider an FX drift-diffusion model across a bent ZnO NR (Figure 3d and Supplementary Note 4). During the FX lifetime $\left(\tau_{\mathrm{FX}}\right)$, FXs excited at incident e-beam position $\left(r=r_{k}\right)$ drift under the graded band gap at a mobility $\mu_{\mathrm{FX}}$ and diffuse with diffusion constant $D_{\mathrm{FX}}$. Figure 6e illustrates the impact of FX drift on a line-fitting analysis of spot- $\mathrm{CL}$ plots in each run, which accounts for both $\delta E_{\mathrm{g}}(0)$ red shift and $a_{c \mathrm{~N} N}$ quench when increasing the NR bending. 1D rate equation of FX spatial distribution $n_{\mathrm{FX}}(r)$ is formulated considering three steps: carrier excitation and relaxation toward band edges to form FX, FX drift-diffusion, and FX recombination (eq 1). On the basis of the idea that the FX center-of-mass reaches the outer edge of a bent NR $(r=D / 2)$ at large $R^{-1}$ where $a_{c c N}$ quenches and negative $\delta E_{\mathrm{g}}(0)$ shift saturates, $a_{c c}\left(R^{-1}\right)$ and $\left[\delta E_{\mathrm{g}}(0)\right]\left(R^{-1}\right)$ are formulated (eqs 2 and 3 ).

$$
0=g_{0} \delta(r)+D_{\mathrm{FX}} \nabla^{2} n_{\mathrm{FX}}(r)-\mu_{\mathrm{FX}} F_{\mathrm{g}} \nabla n_{\mathrm{FX}}(r)-\frac{n_{\mathrm{FX}}(r)}{\tau_{\mathrm{FX}}}
$$

$a_{c C}\left(R^{-1}\right)=a_{c C}\left[1-\left\{\frac{1-\exp \left(-\frac{D / 2}{\left|a_{c C}\right| \mu_{\mathrm{FX}} \tau_{\mathrm{FX}} R^{-1}}\right)}{\frac{D / 2}{\left|a_{c c}\right| \mu_{\mathrm{FX}} \tau_{\mathrm{FX}} R^{-1}}}\right\}\right]$

$$
\left[\delta E_{\mathrm{g}}(0)\right]\left(R^{-1}\right) \propto a_{c \mathrm{c}} \frac{D}{2}\left[\frac{1-\exp \left(-\frac{D / 2}{\left|a_{\mathrm{cC}}\right| \mu_{\mathrm{FX}} \tau_{\mathrm{FX}} R^{-1}}\right)}{\frac{D / 2}{\left|a_{c \mathrm{C}}\right| \mu_{\mathrm{FX}} \tau_{\mathrm{FX}} R^{-1}}}\right]
$$

The $\left(R^{-1}, a_{c c \mathrm{~N}}\right)$ plots in Figure $3 \mathrm{~b}$ are fitted by the $a_{c c}\left(R^{-1}\right)$ curve at $D=0.15 \mu \mathrm{m}$ and $a_{c c}=-1.7 \mathrm{eV}$. The 


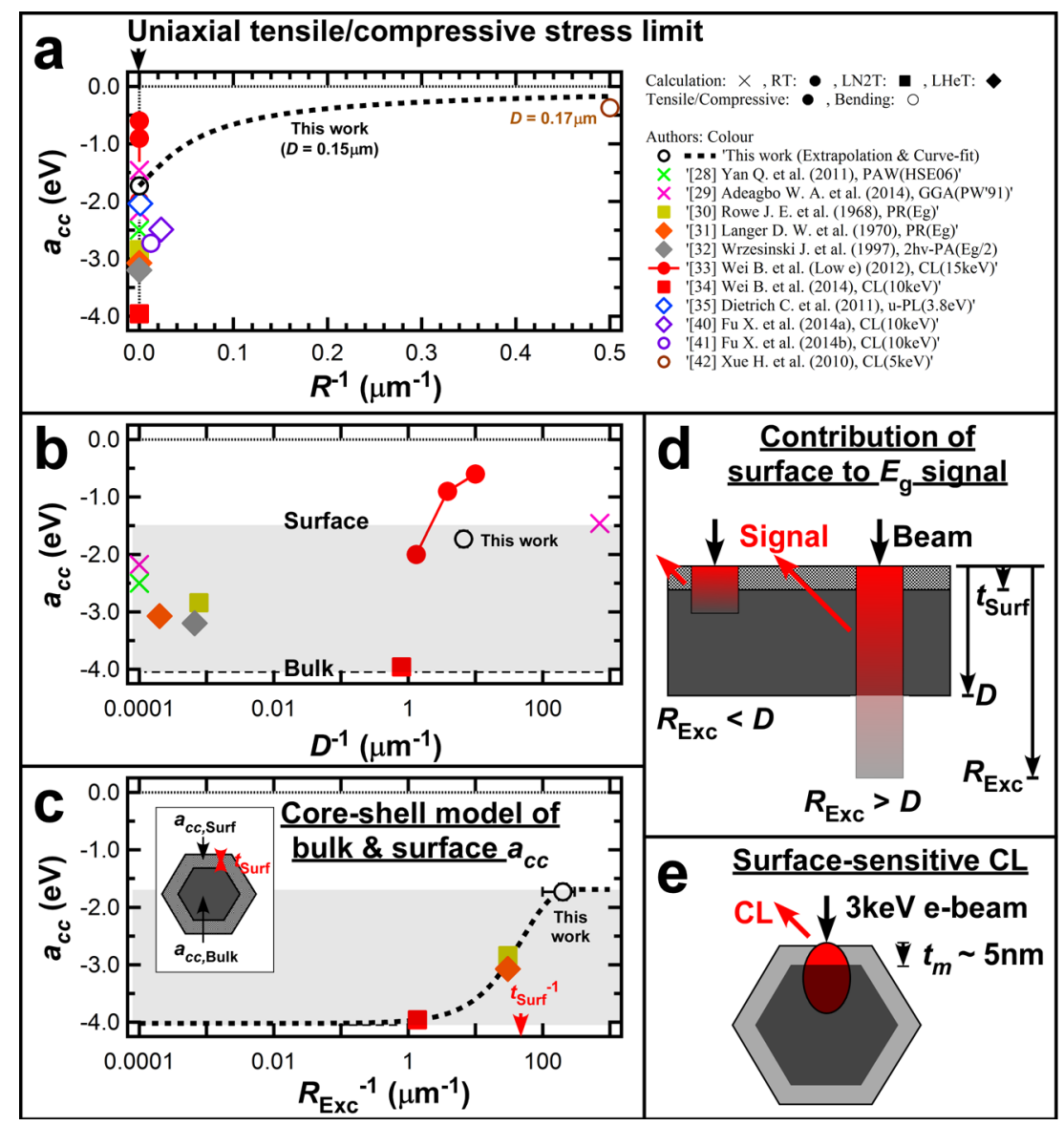

Figure 4. Impact of uniaxial strain gradient and NR surface elasticity on the reported $a_{c c}(\mathrm{eV})$. Data plots from previous reports and this study: (a) $\left(R^{-1}, a_{c c}\right)$, (b) $\left(D^{-1}, a_{c c}\right)$, and (c) $\left(R_{\mathrm{Exc}}{ }^{-1}, a_{c c}\right)$. The plots are categorized as follows. Each report is labeled with its first author's name followed by a measurement method (PR, $2 h v-\mathrm{PA}, \mathrm{PL}$, or $\mathrm{CL}$ ) with its carrier excitation beam energy or a calculation method (PAW or GGA) with its calculation code. Symbol color indicates each research group. The cross marks indicate theoretical calculations. Bulk $D$ values in the calculation ${ }^{28,29}$ are infinite; however, they are plotted as $D=10 \mathrm{~mm}$ for display in panel b. The specimen temperature is indicated by a circle for room temperature $(\sim 300 \mathrm{~K})$, by a square for liquid $\mathrm{N}_{2}$ temperature $(\sim 80 \mathrm{~K})$, and by a rotated square for liquid He temperature $(\sim 10 \mathrm{~K})$. Solid symbols indicate $\mathrm{ZnO}$ under uniaxial tensile or compressive stress, which are plotted at no bending limit: $R^{-1}=0$ in panel a. Empty symbols indicate ZnO under uniaxial bending stress. The $2 h v-\mathrm{PA}$ plot $^{32}$ is omitted in panel $\mathrm{C}$ since $\alpha\left(E_{\mathrm{g}} / 2\right)$ values due to $\mathrm{ZnO}$ defect band DOS are significantly specimen dependent. Panel $\mathrm{c}$ inset describes the cross-sectional view of a $\mathrm{ZnO}$ wire where the surface with thickness $t_{\text {surf }}$ is modeled to have a different elasticity and $a_{c c}$ from the bulk. On the basis of this core-shell model, the $a_{c c}$ trend for $R_{\mathrm{Exc}}<D$ cases is curve-fitted by $a_{\mathrm{cc}}\left(R_{\mathrm{Exc}}{ }^{-1}\right)$ in eqs 5 and 6 (dashed line). (D) $\mathrm{ZnO}$ surface thickness contribution of entire probe surface depth in near-band-edge spectroscopy. Carrier excitation depth $R_{\mathrm{Exc}}$ with respect to $\mathrm{ZnO}$ wire diameter $D$ is considered at two limits. (e) Schematic of our surface-sensitive CL spectroscopy. Surface CL signal from entire CL emission domain (red ellipsoid) is highlighted by the NR lateral growth domain with a thickness of $t_{\mathrm{m}}=5 \pm 2 \mathrm{~nm}$, which is more luminescent than the NR axial growth domain.

fitted $a_{c c}\left(R^{-1}\right)$ curve reproduces the trend of $\left(R^{-1}, a_{c c \mathrm{~N}}\right)$ plots, and the FX diffusion parameter is evaluated to be $\mu_{\mathrm{FX}} \tau_{\mathrm{FX}}=0.41 \pm 0.08 \mu \mathrm{m}^{2} / \mathrm{eV}$ at $T_{\mathrm{NR}}=300 \mathrm{~K}$. Further, the $\left(R^{-1}, \delta E_{\mathrm{g}}(0)\right)$ plots in Figure $3 c$ are fitted by the $\left[\delta E_{\mathrm{g}}(0)\right]\left(R^{-1}\right)$ curve at given parameters $D=0.15 \mu \mathrm{m}$, $a_{c C}=-1.7 \mathrm{eV}$, and $\mu_{\mathrm{FX}} \tau_{\mathrm{FX}}=0.41 \mu \mathrm{m}^{2} / \mathrm{eV}$ and with two fit parameters: an energy offset of $8 \pm 5 \mathrm{meV}$, comparable with a $\delta E_{\mathrm{g}}(0)$ error of $4.1 \mathrm{meV}$, and a proportional factor less than unity. The fitted $\left[\delta E_{\mathrm{g}}(0)\right]\left(R^{-1}\right)$ curve also reproduces the trend of $\left(R^{-1}, \delta E_{\mathrm{g}}(0)\right)$ plots in detail.

Our FX drift parameter is significantly smaller than the reported neutral-donor-bound exciton $\left(D^{0} X\right)$ drift parameter at $T=8 \mathrm{~K}\left(\mu_{\mathrm{Dox}} \tau_{\mathrm{Dox}}=57.6 \mu \mathrm{m}^{2} / \mathrm{eV}\right)$, evaluated by a time-resolved CL (TR-CL) study of a preliminarily bent ZnO MW $\left(D=2.24 \mu \mathrm{m}, R^{-1}=0.0125 \mu \mathrm{m}^{-1}\right){ }^{41}$ This is not surprising since (1) the phonon scattering at elevated temperature may decrease the $\mu_{\mathrm{Fx}}$ and (2) the surface-to-depth of the spot-CL spectroscopy probe is smaller, where the $\tau_{\mathrm{Fx}}$ should be smaller due to nonradiative recombination at the surface, radiative recombination with residual carriers concentrated in the NR lateral growth domain, or something else (Supplementary Note 6). Since the FX drift is so slow in $\mathrm{ZnO}$ at room temperature, it is only observable in a NR under a large strain gradient $d \varepsilon_{c c N} / d r$. Such point bending stress applied to a free-standing NR forms a well-defined uniaxial strain gradient in an entire structure. Observed room-temperature minority carrier drift in the presence of a strain gradient is significant to clarify the carrier conduction path in a 


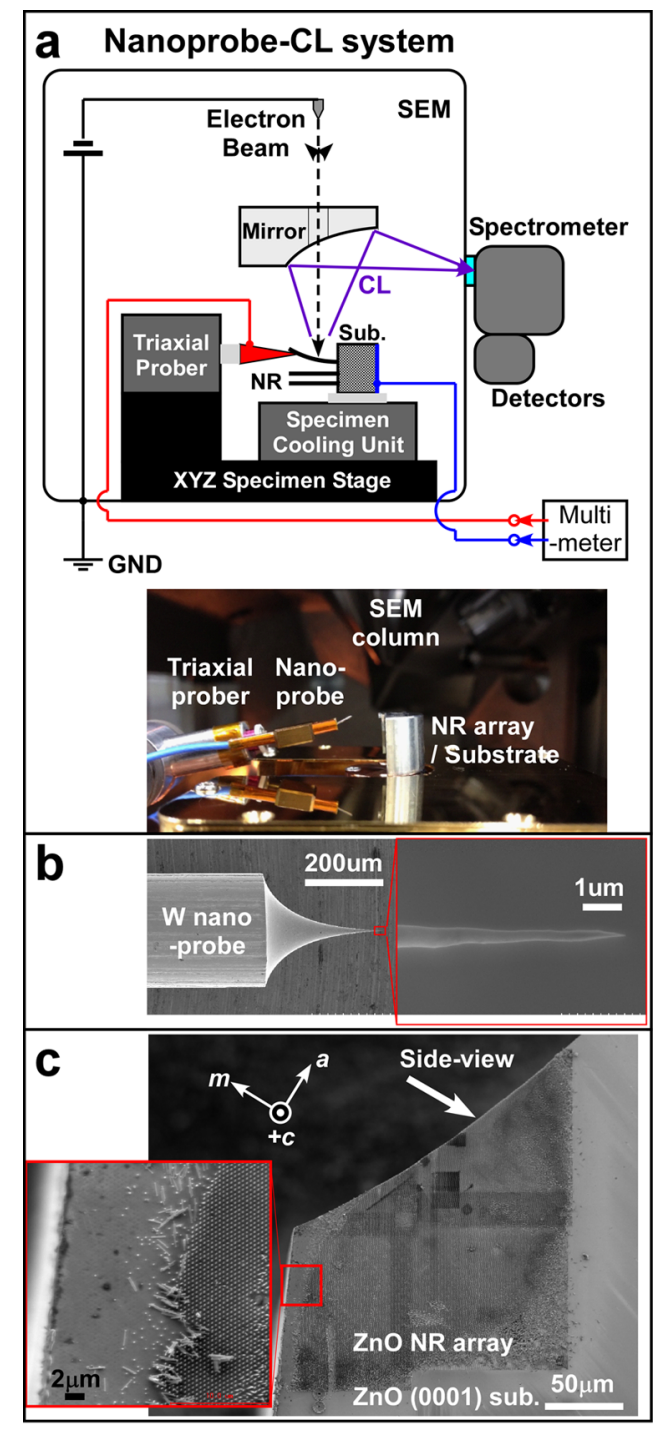

Figure 5. Nanoprobe-CL system and its application to a $\mathrm{ZnO}$ free-standing NR array. (A) Schematic of the nanoprobe-CL system and a photograph of an experimental setup. The system equips a piezoelectric nanomanipulator for a W-nanoprobe indenter and a low-temperature specimen cooling unit $(10-300 \mathrm{~K})$ on an XYZ stage of a SEM-CL system. The $\mathrm{W}$-nanoprobe indenter and specimen are electrically connected with a multimeter to monitor W-nanoprobe contact to a free-standing NR apex. (B) SEM image of an electrochemically etched $W$-nanoprobe indenter. (C) SEM image of a free-standing $\mathrm{ZnO}$ NR array fabricated on a $\mathrm{ZnO}$ (0001) substrate. The substrate is cleaved to load NRs at the edge with a W-nanoprobe indenter, and their deformation is observed by SEM.

device simulation. Simultaneously, it is a principle of novel devices controlling carrier population by external strain gradient.

\section{DISCUSSION}

Factors of $a_{c c}$ Value Scattering in the Literature: Strain Gradients and Surface Elasticity. Here we discuss the scattering origin of reported $a_{c c}$ values of $\mathrm{ZnO}$ wires. Reported $a_{c c}$ values are plotted with inverse wire curvature $R^{-1}$ (uniaxial strain gradient) (Figure 4a), with inverse wire diameter $D^{-1}$ (surface-to-volume ratio) (Figure $4 b$ ), and with inverse probe surface depth $R_{\mathrm{Exc}}(E)^{-1}$ of probe (photon or electron) energy $E$ (probe depth-to-volume ratio) (Figure 4c). Our fit curve $a_{c c}\left(R^{-1}\right)$ is superimposed in Figure $4 \mathrm{a}$, and our result $\left(a_{c c}=-1.7 \mathrm{eV}\right)$ is plotted in Figure $4 \mathrm{~b}$ and c. Previous experimental $a_{c c}$ studies are based on spectroscopy of photoreflectance, two-photon ( $2 h v$-PA) absorption, PL, or $\mathrm{CL}$. Previous $a_{c c}$ calculations are based on generalized gradient approximation (GGA) or the projectoraugmented wave method (PAW), which are plotted in Figure $4 \mathrm{~b}$. When we calculate $a_{c c}$ from reported conduction band $d_{i}(i=1,2)$ and valence band $C_{i}(i=1,2, \ldots)$ deformation potentials ${ }^{29-33}$ of the Pikus-Bir strain Hamiltonian ${ }^{31,46}$ for a wurzite semiconductor $\left(\Gamma_{9 \mathrm{v}}\right.$ valence band symmetry), the reported bulk $\mathrm{ZnO}$ Poisson ratio in c-axis ${ }^{47}\left(v_{c, \text { Bulk }}=0.32\right)$ is used.

$$
a_{\mathrm{cc}}=\left(d_{1}+C_{1}+C_{3}\right)-2 v_{c, \text { Bulk }}\left(d_{2}+C_{2}+C_{4}\right)
$$

The $R_{\mathrm{Exc}}(E)$, illustrated in Figure $4 \mathrm{~d}$, is evaluated assuming that their $\mathrm{ZnO}$ wires are electrically uniform: $R_{\mathrm{Exc}}(E)$ $=\alpha(E)^{-1}$ for the laser beam (PR and PL) using typical NBE optical absorption coefficient ${ }^{48} \alpha(E)$, and $R_{\mathrm{Exc}}(E)=$ $R_{\mathrm{KO}}(E)$ for the electron beam $(\mathrm{CL})$, using the KanayaOkayama range ${ }^{49} R_{\mathrm{KO}}(E)$. Our result is plotted with $R_{\text {Exc }}{ }^{-1}=t_{\mathrm{m}}{ }^{-1}$ in Figure $4 \mathrm{c}$ because NBE CL at $3 \mathrm{keV}$ e-beam incidence $\left(R_{\mathrm{KO}}=97 \mathrm{~nm}\right)$ is dominantly emitted from a luminescent but thin $\left(t_{\mathrm{m}}=5 \pm 2 \mathrm{~nm}\right)$ lateral growth domain of $\mathrm{ZnO} \mathrm{NR}$, as illustrated in Figure $4 \mathrm{e}$ (Supplementary Note 6). Here we do not plot the reference data obtained by area spectroscopies of bent $\mathrm{ZnO}$ wires, since their inaccuracies are obvious (Supplementary Note 1).

Experimental and calculation $\left(R^{-1}, a_{c c}\right)$ plots are shown in Figure $4 a$. They are scattered even at no strain gradient $\left(R^{-1}=0\right)$, and $\left|a_{c c}\right|$ tends to be smaller at larger strain gradient $R^{-1}$. Note that our $a_{c c}\left(R^{-1}\right)$ curve explains the brown plot $^{42}$ that is obtained from $5 \mathrm{keV}$ spot-CL spectroscopy across a bent ZnO NW at RT $(D=$ $0.17 \mu \mathrm{m}$, typically $R=2-4 \mu \mathrm{m}$ from SEM images in Figure 5). We consider that this plot underestimates the $\left|a_{c c}\right|$ due to the FX drift, rather than due to the insufficient in-plane $\mathrm{CL}$ resolution. ${ }^{35}$ Similarly, other $\mu$ $\mathrm{PL}^{35}$ and $\mathrm{CL}^{40-42}$ measurements of bent MWs may underestimate the $\left|a_{c c}\right|$ value as well. The $\left(D^{-1}, a_{c c}\right)$ plots under uniform uniaxial tensile/compressive stress are shown in Figure 4b. The GGA(PW'91) calculation ${ }^{29}$ suggests that NW $a_{c c}$ is smaller than the bulk value (bulk $D$ is infinity but plotted as $D=10 \mathrm{~mm}$ for display), though its quantitative accuracy remains in question. Wei et al. performed CL spectroscopy of tensile NWs using a $15 \mathrm{keV}$ e-beam probe $\left(R_{\mathrm{Exc}}=1.4 \mu \mathrm{m}\right)$ and reported that $\left|a_{c c}\right|$ at small tensile strain decreases with $D^{-1}$ in the sub-micrometer $D$ range. ${ }^{33}$ They attributed it to the elasticity of $\mathrm{ZnO}$ surface reconstruction. ${ }^{33} \mathrm{How}$ ever, $\mathrm{ZnO}(10-10)$ surface reconstruction is in the range of surface depth on the order of $1 \mathrm{~nm} .^{20-22,27}$ 
Such a small volume fraction of surface reconstruction does not significantly affect the strain magnitude of an entire NW in the sub-micrometer $D$ range, unless the surface Young's modulus is at least 2 orders of magnitude larger than the bulk one. Also, their $15 \mathrm{keV}$ e-beam probe is not sensitive to surface reconstruction of sub-micrometer-thick NWs. Thus, the $\left|a_{c c}\right|$ decrease with $D^{-1}$ should originate from some other measurement factors. All other experimental $\left(D^{-1}, a_{c c}\right)$ plots including ours in Figure $4 \mathrm{~b}$ are less scattered than those in Figure 4a. However, their trend is still unclear because wire $D$ in any plot is too large to reveal a surface elasticity-related trend. In contrast, their $\left(R_{\mathrm{Exc}}{ }^{-1}, a_{\mathrm{cc}}\right)$ plots in Figure $4 \mathrm{c}$ exhibit a clear trend that a surface-sensitive measurement with a large $R_{\text {Exc }}{ }^{-1}$ yields a smaller $\left|a_{c c}\right|$. This idea also accounts for purple plots ${ }^{40,41}$ of $10 \mathrm{keV}$ spot-CL spectroscopy $\left(R_{\mathrm{Exc}}=0.73 \mu \mathrm{m}\right)$, located below our dashed black curve in Figure $4 \mathrm{a}$, in terms of the bulk $a_{c c}$ measurement subject to exciton drift. Also, contrary to $\mathrm{ZnO}$ under uniaxial stress, $\mathrm{ZnO}$ under hydrostatic pressure exhibits convergent $a_{\mathrm{p}}$ between -3.5 and $-4.5 \mathrm{eV}$, regardless of the specimen volume $V$ (Figure S3a) and of the measurement surface depth $R_{\mathrm{Exc}}$ (Figure $\mathrm{S} 3 \mathrm{~b}$ ). Note that both $a_{c c}$ and $a_{\mathrm{p}}$, the first $\varepsilon$-derivative of $E_{\mathrm{g}}$ seem insensitive to the $T$, in contrast to the Varsini rule, where $E_{\mathrm{g}}$ decreases with the lattice thermal dilatation. Such a different quantitative consensus between $a_{c c}$ and $a_{\mathrm{p}}$ values suggests that the uniaxial stress affects $\mathrm{ZnO}$ band-gap deformation potential at NR surfaces.

Here we account for the $a_{c c}$ trend in Figure $4 c$ in terms of lattice strain relaxation at the $\mathrm{ZnO}$ side surface under the uniaxial tensile/compressive stress. Since the surface lattice is less constrained than the bulk one, wurzite lattice spacing $d$ is less $\varepsilon_{c c}$ sensitive at the surface, and thus the band gap $E_{\mathrm{g}}$ is less sensitive as well. Therein, any spectroscopy of uniaxially strained $\mathrm{ZnO}$ results in a single $E_{\mathrm{g}}$ peak that contains contributions of a certain surface-to-depth. On the contrary, in the case of a $\mathrm{ZnO}$ crystal of volume $V$ under hydrostatic pressure $P$, since the surface lattice is also constrained as the bulk lattice, the $\mathrm{ZnO}$ crystal is compressed uniformly. Thus, the $\mathrm{d} E_{\mathrm{g}} / \mathrm{d}(\ln V)=a_{\mathrm{p}} \approx-4 \mathrm{eV}$ is purely a bulk response (Figure S3). To evaluate the surface depth of lattice strain relaxation $t_{\text {surf, }}$ we adopt a core-shell approximation of $a_{c c}$ in the bulk $\left(a_{c c, \text { Bulk }}\right)$ and at the surface $\left(a_{c c, \text { Surf }}\right)$. An experimental $a_{c c}$ value is expressed at first approximation by eq 5 , where surface contribution $f_{\text {Surf, }}$ eq 6 , is evaluated by the fraction of surface thickness with respect to the carrier excitation surface-to-depth in wire thickness $D$. We then consider the $R_{\text {Exc }}$ with respect to the $D\left(>t_{\text {Surf }}\right)$ at two limits (Figure $4 \mathrm{~d}$ ): $R_{\mathrm{Exc}}>D$ for ref 33 and $R_{\mathrm{Exc}}<D$ for refs $30-32,34,35,40,41$ and this work.

$$
a_{c c}=a_{c c, \text { Bulk }}\left(1-f_{\text {Surf }}\right)+a_{c c, \text { Surf }} f_{\text {Surf }}
$$

$$
\begin{aligned}
f_{\text {Surf }} \approx & \frac{\int_{0}^{t_{\text {Surf }}} \frac{\mathrm{d} z}{R_{\mathrm{Exc}}} \exp \left(-\frac{z}{R_{\mathrm{Exc}}}\right)}{\int_{0}^{D} \frac{\mathrm{d} z}{R_{\mathrm{Exc}}} \exp \left(-\frac{z}{R_{\mathrm{Exc}}}\right)} \\
= & \frac{1-\exp \left(-\frac{t_{\text {Surf }}}{R_{\mathrm{Exc}}}\right)}{1-\exp \left(-\frac{D}{R_{\mathrm{Exc}}}\right)} \stackrel{R_{\mathrm{Exc}}<D}{\longrightarrow} 1-\exp \left(-\frac{t_{\text {Surf }}}{R_{\mathrm{Exc}}}\right)
\end{aligned}
$$

Wei et al. also reported $a_{c c}=-3.96 \mathrm{eV}$ of tensile MW $(D=1.26 \mu \mathrm{m})$ using a $10 \mathrm{keV}$ e-beam probe $\left(R_{\mathrm{Exc}}=\right.$ $0.73 \mu \mathrm{m}){ }^{34}$ which should be a bulk $a_{c c}$ measurement from this picture. On the contrary, our measurement is the most surface-sensitive $\left(R_{\mathrm{Exc}} \approx 5 \mathrm{~nm}\right)$ among all previous works. PR spectroscopies of bulk $\mathrm{ZnO}^{30,31}$ have intermediate surface sensitivities, giving intermediate $a_{c c}$ values. A curve fit of eqs 5 and 6 to experimental plots in Figure $4 \mathrm{c}$ gives a quantitative estimation of $t_{\text {Surf }}=20 \pm 5 \mathrm{~nm}$ as well as $a_{c c, \text { Bulk }}=-4.0 \pm$ $0.2 \mathrm{eV}$ and $a_{c c, \text { Surf }}=-1.7 \pm 0.2 \mathrm{eV}$.

Lattice strain relaxation at the $\mathrm{ZnO}$ side surface under uniaxial stress is clarified by considering surface and bulk Poisson ratios: $v_{c, \text { Surf }}$ and $v_{c, \text { Bulk. }}$. Poisson ratio $v_{c}$ of $\mathrm{ZnO} \mathrm{NW}$ under c-axial tensile stress is given by $c$-plane biaxial strain $\left(\varepsilon_{a a}\right)$ response to the uniaxial strain $\left(\varepsilon_{c c}>0\right): v_{c} \equiv-\varepsilon_{a a} / \varepsilon_{c c}$. An off-axial interspace $d$ of the hexagonal ( $Z n$ atom) lattice is formulated using wurzite $\mathrm{ZnO}$ lattice constants $(c, a), \varepsilon_{c c}$, and $v_{c}{ }^{18}$

$$
\begin{aligned}
d & =\sqrt{\left\{\frac{c}{2}\left(1+\varepsilon_{c c}\right)\right\}^{2}+\left\{\frac{a}{\sqrt{3}}\left(1-v_{c} \varepsilon_{c c}\right)\right\}^{2}} \\
& \approx d_{0}\left[1+\varepsilon_{c c} \frac{a^{2}}{3 d_{0}^{2}}\left(\frac{3 c^{2}}{4 a^{2}}-v_{c}\right)\right]
\end{aligned}
$$

Since $\mathrm{ZnO} E_{\mathrm{g}}$ decreases with the axial tension, $\varepsilon_{c c}$ originating from the change of the effective lattice interspace $d$, our conclusion [ $\left.\left|a_{c c, \text { Surf }}\right|<\left|a_{c c, \text { Bulk }}\right|\right]$ is attributed to the $\varepsilon_{c c}$ sensitivity of the $d$, smaller at the side surface than in the bulk due to different lattice constraints. These conditions suggest that the $v_{c, \text { Surf }}$ should satisfy $v_{c, \text { Bulk }}(=0.32)<v_{c, \text { Surf }}<3 c^{2} / 4 a^{2}(=1.9)$. In other words, under uniaxial stress along the $c$-axis, $\mathrm{ZnO}$ side surface has a unique Poisson ratio, and thus biaxial strain $\varepsilon_{a a}$ in the $c$-plane is relaxed at nonpolar plane surfaces.

We note that the impact of $\mathrm{ZnO}$ surface reconstruction on the $a_{c c}$, within $1 \mathrm{~nm}$ from NR side surfaces, may be observable by our nanoprobe- $C L$ technique. The $\mathrm{ZnO}$ band gap at surface reconstruction should be different from that of the bulk at no applied stress, which is observable as a separated $C L$ peak if the $\mathrm{ZnO}$ surface is luminescent. However, the NBE CL peak energy is identical to that of bulk $\mathrm{ZnO}$, and the $\mathrm{CL}$ signal from the $\mathrm{ZnO}$ surface reconstruction is not observed (Figure S4c). Thus, the aforementioned longrange surface elasticity effects on $a_{c c}$ are the outcome 
of anisotropic stress and probably not the outcome of the surface reconstruction, which should be revealed by more surface-sensitive near-band-edge spectroscopy based on STM. ${ }^{50-52}$

Nanoprobe-CL Technique Impacts in This Study and Its Potential Applications. The nanoprobe- $\mathrm{CL}$ technique takes advantages of a model nanostructure loaded by an in situ nanoprobe indenter, where it is essential to evaluate local $E_{\mathrm{g}}\left(\varepsilon_{i j}\right)$ response to strain $\varepsilon_{i j}$ with specific tensor components at a regulated surface depth and at controlled strain gradient. This work focused on a mechanically robust free-standing $\mathrm{ZnO}$ NR under point bending stress. We revealed the $E_{\mathrm{g}}$ response to diagonal uniaxial $\varepsilon_{z z}$ (and biaxial $\varepsilon_{a a}$ ) components and investigated minority carrier transport induced by its strain gradient $\mathrm{d} \varepsilon_{z z} / \mathrm{d} r$ due to uniaxial bending stress. We also found small surface $E_{\mathrm{g}}$ response (small $a_{c c}$ ) and attributed it to relaxed biaxial $\varepsilon_{a a}$ at side surfaces. Similarly, the nanoprobe-CL technique will reveal an $E_{\mathrm{g}}$ response to nondiagonal shear $\varepsilon_{x z}$ components, by modeling a free-standing pillar under axial twisting stress load. Such comprehensive studies will fully reveal an $E_{\mathrm{g}}\left(\varepsilon_{i j}\right)$ response to strain tensor $\varepsilon_{i j}$ with arbitrary tensor components and minority carrier population driven by a spatial gradient of $\varepsilon_{i j}(x, y, z)$ in a structure. The nanoprobe-CL system is also a potential platform for electrical measurements ${ }^{53}$ under mechanical stress (Figure 5a), which may explore the effective mass $m_{k l}\left(\varepsilon_{i j}\right)$ response to applied $\varepsilon_{i j}$ from the carrier mobility evaluated. ${ }^{54}$ Further, the nanoprobe- $\mathrm{CL}$ technique is also available at low temperature (Figure S5) to enhance the CL signal of indirect-gap materials (e.g., $\mathrm{Si}, \mathrm{Ge}){ }^{55}$ This opens up applications for other materials, since such freestanding pillar structures of various semiconductors are available now both from single-crystalline NW growth (bottom-up) and from lithography of single crystals $^{56}$ (top-down). Knowledge available from the nanoprobe- $\mathrm{CL}$ technique allows us to develop reliable device simulations starting from strain distribution $\varepsilon_{i j}(x, y, z)$ in a structure. Also, the nanoprobe-CL technique may investigate strain-dependent optical and electrical properties of various nanostructures on Si (e.g., nanodot ${ }^{52}$ or thin-film ${ }^{55}$ structures), which is also informative for improving nanodevices, such as FETs or LDs.

\section{CONCLUSIONS}

We demonstrated SEM-CL nanospectroscopy of individual ZnO free-standing NRs $(D=150 \mathrm{~nm})$ under in situ nanomanipulation at the highest in-plane resolution and surface sensitivity (nanoprobe-CL). A freestanding NR under point bending stress is studied for its mechanical robustness, to investigate band-gap response to pure uniaxial strain and strain-gradient impact on minority carrier populations both in wide ranges. SEM observation of the in situ NR bending deformation cycle reveals the NR elasticity limit $\left(\varepsilon_{c c}=\right.$ 0.04). Uniaxial band-gap deformation potential is evaluated to be $a_{c c}=-1.7 \mathrm{eV}$ to the limit of no uniaxial strain gradient, whereas $a_{c c}$ quenches with increasing gradient, which is attributed to free-exciton drift across a bent NR. Surface-sensitive CL measurements suggest that the discrepancy from reported bulk $a_{c c}=-4 \mathrm{eV}$ may originate from lattice strain relaxation at NR side surfaces. The nanoprobe- $\mathrm{CL}$ technique potentially has diverse applications to other materials, structures, mechanical systems, and device simulation, all of which will advance strain-engineered nanodevices, such as FETs or LDs.

\section{METHODS}

The nanoprobe-CL system (Figure $5 \mathrm{a}$ ) is developed based on a SEM-CL spectroscope and equips a XYZ specimen stage with a liquid $\mathrm{N}_{2} / \mathrm{He}$ cooling line $\left(T_{\text {Stage }}=10-300 \mathrm{~K}\right)$ and a piezoelectric nanomanipulator of an electrochemically etched $\mathrm{W}$-nanoprobe indenter (Figure $5 \mathrm{~b}$ ). In this study, a primary e-beam of $3 \mathrm{keV}$ and $2.4 \mathrm{nA}$ is used to observe NR bending deformation in situ by SEM and to probe local $E_{\mathrm{g}}$ as a free-exciton peak energy $\left(E_{\mathrm{FX}}\right)$ in an NBE CL spectrum. In-plane resolutions of SEM and CL imaging are 7.7 and $64 \mathrm{~nm}$, respectively. The former is evaluated by sigmoidal fitting of a secondary electron intensity profile across a $\mathrm{ZnO} \mathrm{NR}$, and the latter is from our previous study. ${ }^{58} \mathrm{CL}$ wavelength resolution in this study is $0.52 \mathrm{~nm}$ ( $4.3 \mathrm{meV}$ for ZnO NBE emission).

A free-standing ZnO NR array is grown on a $\mathrm{ZnO}(0001)$ substrate by an "area-selective homoepitaxy" in a low-temperature $\mathrm{ZnO}$ precursor aqueous solution, ${ }^{57}$ so that each NR bottom is rigidly fixed until its fracture. A trigonal array of circular growth windows with a diameter of $D_{\mathrm{w}}=150 \mathrm{~nm}$ and lattice size of $a=500 \mathrm{~nm}$ is fabricated by electron beam lithography of a $300 \mathrm{~nm}$ thick polymethyl-methacrylate (PMMA) film spincoated on a single-crystalline $\mathrm{ZnO}(0001)$ substrate grown by a hydrothermal method. Each free-standing ZnO NR is grown at the corresponding growth window in the precursor aqueous solution, which consists of a $+c$ top-plane and six $m:\{1-100\}$ side planes, with an averaged diameter of $D=0.15 \mu \mathrm{m}$ and length of $L=1.4 \mu \mathrm{m}$. In this study, NR lateral growth thickness is suppressed ( $t_{\mathrm{m}}=5 \pm 2 \mathrm{~nm}$ ) with respect to NR growth window size so that the NR diameter is uniform along its axis. Simultaneously, surface sensitivity of the $3 \mathrm{keV} \mathrm{CL}$ probe is enhanced by the luminescent $\mathrm{NR}$ lateral growth domain $\left(R_{\mathrm{Exc}} \approx t_{\mathrm{m}}\right)$ (Supplementary Note 6). After the PMMA film removal, the $\mathrm{ZnO}$ substrate is then cleaved so that NRs in the vicinity $(<a)$ of the cleaved edge are accessible by $\mathrm{W}$-nanoprobe and observable by SEM (Figure $5 \mathrm{c}$ ). In order not to observe $\mathrm{CL}$ of the $\mathrm{ZnO}$ substrate surface by e-beam, the substrate surface is tilted behind from PE beam axis by about $1^{\circ}$ (Figure $5 a$, inset photograph).

In our in situ NR bending setup, a free-standing ZnO NR bottom is naturally clamped on the substrate by homoepitaxy and the NR apex is deflected laterally by a W-nanoprobe indenter at a speed of $\mathrm{d} u(t) / \mathrm{d} t=1 \times 10^{2} \mathrm{~nm} / \mathrm{s}$. A sequence of $\mathrm{NR}$ bending deformation cycles is recorded as corresponding SEM images, deflecting the NR apex laterally toward the $<11-20\rangle: a$ direction (Figure 6a). Such a situation is described by Euler-Bernoulli beam theory: a continuum mechanics of a free-standing beam deflected laterally by a point load

$F$ (Figure $6 b$ ). An internal strain of a bent NR distributes so that 


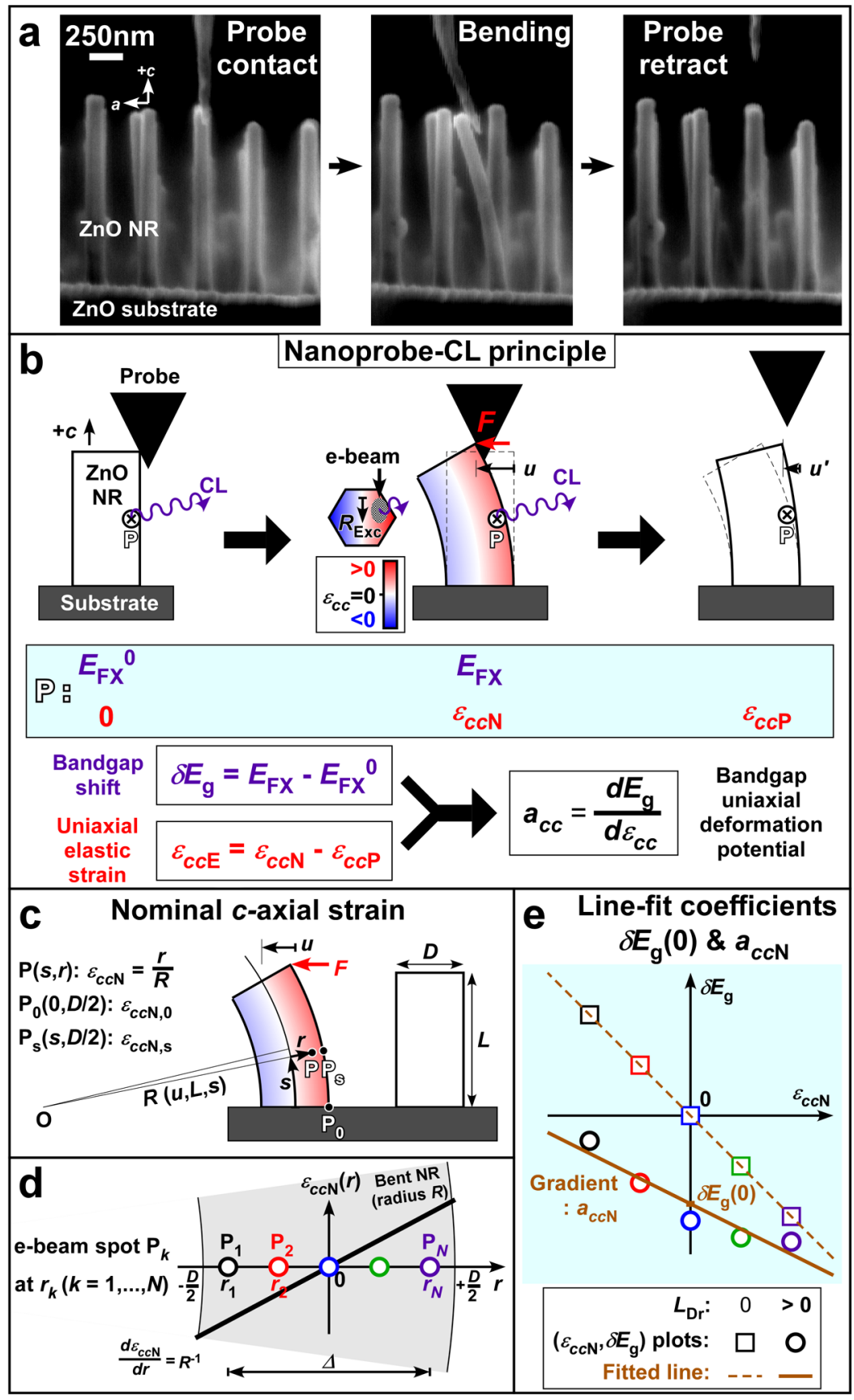

Figure 6. Principles of the nanoprobe- $\mathrm{CL}$ technique: measurement and data analysis using ZnO free-standing NRs. (a) Sequential SEM images of a free-standing ZnO NR under a bending deformation cycle, which consists of "probe contact", "bending", and "probe retract" steps. The NR apex is deflected laterally by manipulating a W-nanoprobe indenter toward the $a:<11-20>$ direction. (b) A principle of nanoprobe-CL measurement under the bending deformation cycle. An apex of a NR with length $L$ and diameter $D$ is deflected by $u$ by a lateral load $F$ in the "bending" step. An unloaded NR exhibits a residual deflection $u^{\prime}$ in the "probe retract" step, due to the plastic deformation. Local uniaxial (c-axis) strain of a bent NR $\left(\varepsilon_{c c}\right)$ is illustrated by a blue-white-red color bar of $\varepsilon_{c c}$ and a neutral plane $\left(\varepsilon_{c c}=0\right)$ present in the middle of the NR is white. An electron beam (e-beam) is spotted at arbitrary position $\mathrm{P}(s, r)$, and local band gap $E_{\mathrm{g}}$ is probed by a free-exciton (FX) peak energy $\left(E_{\mathrm{FX}}\right)$ in a near-band-edge $C L$ spectrum. The strain-induced band-gap shift at $\mathrm{P}(s, r)$ is evaluated by a peak energy shift due to $\mathrm{NR}$ bending $\left(\delta E_{\mathrm{g}}=E_{\mathrm{FX}}-E_{\mathrm{FX}}{ }^{0}\right)$. (c) Schematic that describes an analytical calculation of NR bending curvature $R(u, L, s)$ and nominal strain $\varepsilon_{c c N}=r / R$ at position $\mathrm{P}(s, r)$. The $\varepsilon_{c c N}$ at specific positions $\mathrm{P}_{0}(0, D / 2)$ and $\mathrm{P}_{s}(s, D / 2)$ are labeled $\varepsilon_{c c N, 0}$ and $\varepsilon_{c c N, s}$ respectively. A plastic strain $\varepsilon_{c c P}$ in the "probe retract" step and an elastic strain $\varepsilon_{c C E}$ in the "bending" step at $\mathrm{P}(s, r)$ are evaluated by substituting residual $u^{\prime}$ deflection and net deflection $u-u^{\prime}$ into $u$ of $R(u, L, s)$. (d) Schematic illustration of spot-CL spectroscopy in a "run", where the e-beam is spotted at each $\mathrm{P}_{k}\left(r_{k}, s\right)(k=1, \ldots, N)$ consecutively across a bent NR, indicated by a colored open circle. The $\varepsilon_{c c N}(r)$ curve at a strain gradient $R^{-1}$ is superimposed. (e) Schematic line-fitting analysis of spot-CL data plots $\left(\varepsilon_{c \mathrm{cN}}, \delta E_{\mathrm{g}}\right)$, obtained at each "run". Nominal parameters of interest, $\delta E_{\mathrm{g}}(0)$ and $a_{c c N}$, are evaluated from line-fitting coefficients. Plots at $L_{\mathrm{Dr}}>0$ (open circles) and plots at $L_{\mathrm{Dr}}=0$ (open squares) are depicted to discuss the impact of FX drift in Figure 3. 
it realizes a minimum elastic energy; a ZnO NR is uniaxially strained along its $c$-axis and local c-axial strain $\varepsilon_{c c}$ is positive and negative at the outer and inner side from a central neutral plane, respectively. A nominal $\varepsilon_{c c N}$ and its spatial gradient $\mathrm{d} \varepsilon_{c \mathrm{cN}} / \mathrm{d} r$ at position $\mathrm{P}(s, r)$ are explicitly evaluated using a rectangular NR cross-section approximation. Here, the $R(u, L, s)$ is a local NR curvature radius (eqs 8 and 9 , Figure $6 c$ ). The accuracy of the rectangular approximation is confirmed $(< \pm 5 \%)$ by finiteelement calculations of Chen et al. ${ }^{23}$

$$
\begin{gathered}
\varepsilon_{c \mathrm{cN}}\left(R^{-1}, r\right)=\frac{r}{R}\left(-\frac{D}{2}<r<\frac{D}{2}\right) \\
\frac{\mathrm{d}}{\mathrm{d} r} \varepsilon_{c \mathrm{NN}}=[R(u, L, s)]^{-1} \approx \frac{3(L-s) u}{L^{3}}
\end{gathered}
$$

Some specific $\varepsilon_{c c N}$ values are renamed for convenience; $\varepsilon_{c c N, 0}=$ $u\left(3 D / 2 L^{2}\right)$ at $\mathrm{P}_{0}(0, D / 2)$ and $\varepsilon_{c c N, s}$ at $\mathrm{P}_{s}(s, D / 2)$. The former is a maximum strain throughout the NR, which describes a NR bending magnitude. The latter defines a uniaxial strain range available at a height $s$ of interest. After retracting the nanoprobe, the NR apex may exhibit a residual deflection $u^{\prime}$ due to its plastic deformation (Figure $6 \mathrm{~b}$ ). This allows us to evaluate a plastic strain, $\varepsilon_{C C P}$, and an elastic strain, $\varepsilon_{C C E}$, at each $C L$ measurement position. These $\varepsilon_{C C P}$ and $\varepsilon_{C C E}$ are quantitative in the "elastic" regime, where a stress concentration is negligible (Figure 3a).

$$
\varepsilon_{c C E} \equiv \varepsilon_{c C N}-\varepsilon_{c C P}
$$

In nanoprobe-CL measurements, the focused e-probe for $\mathrm{CL}$ excitation is either spotted (spot-CL spectroscopy) or scanned (area-CL spectroscopy) on a ZnO NR. Spot-CL spectroscopy is intensively performed in order to obtain $\left(\varepsilon_{c c N}, \delta E_{\mathrm{g}}\right)$ plots across a bent ZnO NR of local curvature $R^{-1}$. Each PE beam spot position $\left(s, r_{k}\right)$ in each run is labeled as $\mathrm{P}_{k}(k=1,2, \ldots, N ; N=4$ or 5$)$ (Figure $6 \mathrm{~d}$ ). The $\delta E_{\mathrm{g}}$ at position $\mathrm{P}(s, r)$ is defined as an energy gap between an FX peak at $\mathrm{P}$ on a bent NR $\left[E_{\mathrm{FX}}\right]$ and that on an unbent $N R\left[E_{\mathrm{FX}}{ }^{0}\right]$ (eq 11). Each of them is explicitly determined by Gaussian curve fitting of a FX peak within its fwhm. Here, the $E_{\mathrm{FX}}{ }^{0}$ is measurement position dependent, which is investigated by spot-CL spectroscopy on a typical unbent NR beforehand (Supplementary Note 6 and Figure S4). This is to avoid possible e-beam dose effects on obtained $a_{c \mathrm{cN}}$, such as e-beam-induced activation of a hydrogen donor in $\mathrm{ZnO}$.

$$
\delta E_{\mathrm{g}}\left(\varepsilon_{c \mathrm{cN}}\left(R^{-1}, r\right)\right)=E_{\mathrm{FX}}\left(\varepsilon_{c \mathrm{NN}}\left(R^{-1}, r\right)\right)-E_{\mathrm{FX}}^{0}
$$

Here we consider a Taylor expansion of $\delta E_{\mathrm{g}}\left(\varepsilon_{c c \mathrm{~N}}\right)$ at around $\varepsilon_{c c N}\left(R^{-1}, 0\right)=0$ (eqs 12 and 13). Parameters of our concern, strain-gradient-induced $E_{\mathrm{FX}}$ baseline shift, $\delta E_{\mathrm{g}}(0)$, and $c$-axial band-gap deformation potential, $a_{c c N}$, are zeroth- and firstorder derivatives of $\delta E_{\mathrm{g}}\left(\varepsilon_{c \mathrm{cN}}\right)$ at $\varepsilon_{c \mathrm{cN}}=0$, respectively. The $\delta E_{\mathrm{g}}(0)$ and $a_{c \mathrm{NN}}$ at each run of spot-CL spectroscopy are determined by the line-fitting of the $\left(\varepsilon_{c c \mathrm{~N}}, \delta E_{\mathrm{g}}\right)$ plots and resulting offset and gradient, respectively (eq 12 and Figure 6e).

$$
\begin{gathered}
\delta E_{\mathrm{g}}\left(\varepsilon_{c \mathrm{cN}}\left(R^{-1}, r\right)\right)=\delta E_{\mathrm{g}}(0)+a_{c \mathrm{NN}} \varepsilon_{c \mathrm{NN}}+\mathrm{O}\left(\left(\varepsilon_{c c \mathrm{~N}}\right)^{2}\right) \\
\delta E_{\mathrm{g}}(0) \equiv \delta E_{\mathrm{g}}\left(\varepsilon_{c \mathrm{cN}}\left(R^{-1}, 0\right)\right)
\end{gathered}
$$

The deformation potential due to the elastic strain, $a_{c C E}$, is also evaluated using eq 14 . At no bending limit $(u \rightarrow 0)$, there is no strain gradient $\left(R^{-1} \rightarrow 0\right)$ and NR deformation is fully elastic $\left(\varepsilon_{c C E}=\varepsilon_{c C N}\right)$, where $a_{c C N}$ and $a_{c C E}$ converge to $a_{c C}$. This $a_{c C}$ is of interest and is comparable with $a_{c c}$ from other publications obtained under uniaxial tensile or compressive stress.

$$
\frac{a_{\mathrm{CCE}}}{a_{\mathrm{CCN}}} \approx \frac{\varepsilon_{\mathrm{CCN}, \mathrm{s}}}{\varepsilon_{\mathrm{CCE}, \mathrm{s}}}=\frac{\varepsilon_{\mathrm{CCN}, 0}}{\varepsilon_{\mathrm{CCE}, 0}} \stackrel{u \rightarrow 0}{\longrightarrow} 1
$$

In this study, $\delta E_{g}(0)$ and $a_{c c N}$ are found to depend on the local strain gradient: $\mathrm{d} \varepsilon_{c c \mathrm{~N}} / \mathrm{d} r=R^{-1}$ (Figure $3 \mathrm{~b}$ and $\mathrm{c}$ ). For further discussion on the NR bending effects, band-gap gradient $F_{\mathrm{g}}$ is formulated at the elastic regime (eq 15). Local $\left(\varepsilon_{c c}, \delta E_{\mathrm{g}}\right)$ plots without FX drift $\left[L_{D r}=0\right.$ (open squares)] or with $\mathrm{FX}$ drift $\left[L_{\mathrm{Dr}}>0\right.$ (open circles)] are depicted in Figure 6e, which shows the impact of FX drift on the line-fitting analyses of spot-CL data in each run.

$$
F_{\mathrm{g}}=\frac{\partial\left(\delta E_{\mathrm{g}}\left(\varepsilon_{c \mathrm{cN}}\right)\right)}{\partial r}=\frac{\partial\left\{\delta E_{\mathrm{g}}\left(\varepsilon_{c \mathrm{cN}}\right)\right\}}{\partial \varepsilon_{c \mathrm{~N}}} \frac{\partial\left(\varepsilon_{c \mathrm{NN}}\left(R^{-1}, r\right)\right)}{\partial r}=\frac{a_{c \mathrm{~N}}}{R}
$$

Errors of parameters available from the nanoprobe- $C L$ technique are summarized. The $\varepsilon_{c \mathrm{~N}, 0}$ (or $\varepsilon_{c \mathrm{CP}, 0}$ ) error $( \pm 0.0009)$ and $R^{-1}$ error $( \pm 0.012)$ in observing NR deformation originate from SEM resolution. A monotonic $X Y Z$ specimen stage drift with respect to e-beam and $W$-nanoprobe drift with respect to the NR are evaluated by the corresponding SEM image shifts before and after the CL spectroscopy (see Figure 5a). The former appears as a major factor of $\varepsilon_{C \mathrm{CN}}$ (or $\varepsilon_{\mathrm{CCE}}$ ) error, and the error bar length is determined by the expected spot position, which is evaluated by SEM image shifts during a "run" of sequential spot$\mathrm{CL}$ spectroscopy. The latter was negligible throughout this study. The error of $\delta E_{\mathrm{g}}$, such as $\delta E_{\mathrm{g}}\left(\varepsilon_{c c \mathrm{~N}}\right)$ and $\delta E_{\mathrm{g}}(0)$, originates from statistical $E_{\mathrm{FX}}{ }^{0}$ differences among NRs (4.1 meV, NR $\left.n=20\right)$ rather than $E_{\mathrm{FX}}{ }^{0}(s, r)$ fluctuation within a NR along the $r$-axis (0.9 meV in Figure S4e) or Gaussian-fitting error of the NBE CL peak energy $(0.4 \mathrm{meV})$ : the $E_{\mathrm{FX}}{ }^{0}$ difference between a NR of interest and the typical NR in Figure S4. Error bars of $a_{\mathrm{ccN}}$ (and $\left.a_{c \mathrm{CE}}\right)$ are determined by the sum of their errors due to the monotonic $X Y Z$ stage drift and their errors due to the line-fitting of $\left(\varepsilon_{c c N}, \delta E_{\mathrm{g}}\right)$ plots. The statistical $E_{\mathrm{FX}}{ }^{0}$ difference does not affect the accuracy of the obtained $a_{c c \mathrm{~N}}$, the first derivative of $\delta E_{\mathrm{g}}$. Any effect by quantum confinement energy is ignored since the $\mathrm{FX}$ Bohr radius $\left(a_{\mathrm{B}}=1.35 \mathrm{~nm}\right)^{44}$ is much smaller than the NR diameter $(D=150 \mathrm{~nm})$.

Conflict of Interest: The authors declare no competing financial interest.

Supporting Information Available: Supplementary Figures S1-S5 and Supplementary Notes 1-7 are available free of charge via the Internet at http://pubs.acs.org.

Acknowledgment. K.W. and J.V. conceived this work during the joint project led by Y.W. and J.V. R.E. fabricated the specimen under the supervision of J.V. K.W. designed and developed the measurement system, the experimental setup, and the data analyses based on the theoretical modeling. K.W. performed the measurements with the assistance of Y.W. and T.N. T.S. joined some discussions. All authors have given approval to the manuscript. This work was supported by JSPS KAKENHI (Grant Numbers 23760022 and 26790046) and JSPS-HAS Bilateral Joint Research Projects.

\section{REFERENCES AND NOTES}

1. Bardeen, J.; Shockley, W. Deformation Potentials and Mobilities in Non-Polar Crystals. Phys. Rev. 1950, 80, 72-80.

2. Niquet, Y.-M.; Delerue, C.; Krzeminski, C. Effects of Strain on the Carrier Mobility in Silicon Nanowires. Nano Lett. 2012, 12, 3545-3550.

3. Silver, M.; O'Reilly, E. P. Gain and Radiative Current Density in InGaAs/InGaAsP Lasers with Electrostatically Confined Electron States. IEEE J. Quantum Electron. 1994, 30, 547-553.

4. Signorello, G.; Karg, S.; Björk, M. T. Tuning the Light Emission from GaAs Nanowires over 290 meV with Uniaxial Strain. Nano Lett. 2013, 13, 917-924.

5. Feng, J.; Qian, X.; Huang, C.-W.; Li, J. Strain-Engineered Artificial Atom as a Broad-Spectrum Solar Energy Funnel. Nat. Photonics 2012, 6, 866-872.

6. Wang, Z.-L. Nanopiezotronics. Adv. Mater. 2007, 19, 889-892.

7. Thomas, D. G. The Exciton Spectrum of Zinc Oxide. J. Phys. Chem. Solids 1960, 15, 86-96.

8. Christman, J. A.; Woolcott, R. R., Jr.; Kingon, A. I.; Nemanich, R. J. Piezoelectric Measurements with Atomic Force Microscopy. Appl. Phys. Lett. 1998, 73, 3851-3853.

9. Bates, C. H.; White, W. B.; Roy, R. New High-Pressure Polymorph of ZnO. Science 1962, 137, 993. 
10. Mang, A.; Reimann, K.; Rubenacke, St. Bandgaps, CrystalField Splitting, Spin-Orbit Coupling, and Exciton Binding Energies in ZnO Under Hydrostatic Pressure. Solid State Commun. 1995, 94, 251-254.

11. Segura, A.; Sans, J. A.; Manjon, F. J.; Munoz, A.; HerreraCabrera, M. J. Optical Properties and Electronic Structure of Rock-Salt ZnO under Pressure. Appl. Phys. Lett. 2003, 83, 278-280.

12. Shan, W.; Walukiewicz, W.; Ager, J. W., III; Yu, K. M.; Zhang, Y.; Mao, S. S.; Kling, R.; Kirchner, C.; Waag, A. PressureDependent Photoluminescence Study of ZnO Nanowires. Appl. Phys. Lett. 2005, 86, 153117.

13. Chen, S. J.; Liu, Y. C.; Shao, C. L.; Xu, C. S.; Liu, Y. X.; Wang, L.; Liu, B. B.; Zou, G. T. Photoluminescence of Wurzite ZnO under Hydrostatic Pressure. J. Appl. Phys. 2006, 99, 066102.

14. Yadav, S. K.; Sadowski, T.; Ramprasad, R. Density Functional Theory Study of $\mathrm{Zn} X(X=0, \mathrm{~S}, \mathrm{Se}, \mathrm{Te})$ under Uniaxial Strain. Phys. Rev. B 2010, 81, 144120.

15. Li, S.; Jiang, Q.; Yang, G. W. Uniaxial Strain Modulated Band Gap of ZnO Nanostructures. Appl. Phys. Lett. 2010, 96, 213101.

16. Kulkarni, A. J.; Zhou, M.; Sarasamak, K.; Limpijumnong, S. Novel Phase Transformation in ZnO Nanowires under Tensile Loading. Phys. Rev. Lett. 2006, 97, 105502.

17. Agrawal, R.; Peng, B.; Espinosa, H. D. ExperimentalComputational Investigation of ZnO Nanowires Strength and Fracture. Nano Lett. 2009, 9, 4177-4183.

18. Agrawal, R.; Peng, B.; Gdoutos, E. E.; Espinosa, H. D. Elasticity Size Effects in ZnO Nanowires - A Combined Experimental-Computational Approach. Nano Lett. 2008, 8, 3668-3674.

19. He, M.-R.; Xiao, P.; Zhao, J.; Dai, S.; Ke, F.; Zhu, J. Quantifying the Defect-Dominated Size Effect of Fracture Strain in Single Crystalline ZnO Nanowires. J. Appl. Phys. 2011, 109, 123504.

20. Xu, F.; Qin, Q.; Mishra, A.; Gu, Y.; Zhu, Y. Mechanical Properties of ZnO Nanowires under Different Loading Modes. Nano. Res. 2010, 3, 271-280.

21. Stan, G.; Ciobanu, C. V.; Parthangal, P. M.; Cook, R. F. Diameter-Dependent Radial and Tangential Elastic Moduli of ZnO Nanowires. Nano Lett. 2007, 7, 3691-3697.

22. Chen, C. Q.; Shi, Y.; Zhang, Y. S.; Zhu, J.; Yan, Y. J. Size Dependence of Young's Modulus in ZnO Nanowires. Phys. Rev. Lett. 2006, 96, 075505.

23. Chen, C. Q.; Zhu, J. Bending Strength and Flexibility of $\mathrm{ZnO}$ Nanowire. Appl. Phys. Lett. 2007, 90, 043105.

24. Hoffmann, S.; Ostlund, F.; Michler, J.; Fan, H.-J.; Zacharias, M.; Christiansen, S. H.; Ballif, C. Fracture Strength and Young's Modulus of $\mathrm{ZnO}$ Nanowires. Nanotechnology 2007, 18, 205503

25. Ni, H.; Li, X.-D. Young's Modulus of ZnO Nanobelts Measured Using Atomic Force Microscopy and Nanoindentation Techniques. Nanotechnology 2006, 17, 3591-3597.

26. Bai, X. D.; Gao, P. X.; Wang, Z.-L.; Wang, E.-G. Dual-Mode Mechanical Resonance of Individual ZnO Nanobelts. Appl. Phys. Lett. 2003, 82, 4806-4808.

27. He, M.-L.; Yu, R.; Zhu, J. Subangstrom Profile Imaging of Relaxed ZnO (10-10) Surfaces. Nano Lett. 2012, 12, 704-708.

28. Yan, Q.; Rinke, P.; Winkelnkemper, M.; Qteish, A.; Bimberg, D.; Scheffler, M.; Van de Walle, C. G. Strain Effects and Band Parameters in $\mathrm{MgO}, \mathrm{ZnO}$, and CdO. Appl. Phys. Lett. 2012, 26, 152105.

29. Adeagbo, W. A.; Thomas, S.; Nayak, S. K.; Ernst, A.; Hergert, W. First-Principle Study of Uniaxial Strained and Bent ZnO Wires. Phys. Rev. B 2014, 89, 195135.

30. Rowe, J. E.; Cardona, M.; Pollak, F. H. Valence Band Symmetry and Deformation Potentials of ZnO. Solid State Commun. 1968, 6, 239-242.

31. Langer, D. W.; Euwema, R. N. Spin Exchange in Excitons, the Quasicubic Model and Deformation Potentials in II-VI Compounds. Phys. Rev. B 1970, 2, 4005-4022.

32. Wrzesinski, J.; Fröhlich, D. Two-Photon and Three-Photon Spectroscopy of ZnO under Uniaxial Stress. Phys. Rev. B 1997, 56, 13087-13093.
33. Wei, B.; Zheng, K.; Ji, Y.; Zhang, Y.; Zhang, Z.; Han, X. SizeDependent Bandgap Modulation of ZnO Nanowires by Tensile Strain. Nano Lett. 2012, 12, 4595-4599.

34. Wei, B.; Ji, Y.; Han, X.; Zhang, Z.; Zou, J. Variation of Exciton Emissions of ZnO Whiskers Reversibly Tuned by Axial Tensile Strain. Opt. Exp. 2014, 22, 4000-4005.

35. Dietrich, C. P.; Lange, M.; Klüpfel, F. J.; von Wenckstern, H.; Schmidt-Grund, R.; Grundmann, M. Strain Distribution in Bent ZnO Microwires. Appl. Phys. Lett. 2011, 98, 031105.

36. Xu, S.; Guo, W.; Du, S.; Loy, M. M. T.; Wang, N. Piezotronic Effects on the Optical Properties of ZnO Nanowires. Nano Lett. 2012, 12, 5802-5807.

37. Han, X.; Kou, L.; Lang, X.; Xia, J.; Wang, N.; Qin, R.; Lu, J.; Liao, Z.; Zhang, X.; Shan, X.; et al. Electronic and Mechanical Coupling in Bent ZnO Nanowires. Adv. Mater. 2009, 21, 4937-4941.

38. Han, X.; Kou, L.; Zhang, Z.; Zhang, Z.; Zhu, X.; Xu, J.; Liao, Z.; Guo, W.; Yu, D. Strain-Gradient Effect on Energy Bands in Bent ZnO Microwires. Adv. Mater. 2012, 24, 4707-4711.

39. Liao, Z.-M.; Wu, H.-C.; Fu, Q.; Fu, X.; Zhu, X.; Xu, J.; Shvets, I. V.; Zhang, Z.; Guo, W.; Leprince-Wang, Y.; et al. Strain Induced Exciton Fine-Structure Splitting and Shift in Bent ZnO Microwires. Sci. Rep. 2012, 2, 452.

40. Fu, X.; Su, C.; Fu, Q.; Zhu, X.; Zhu, R.; Liu, C.; Liao, Z.-M.; Xu, J.; Guo, W.; Feng, J.; et al. Tailoring Exciton Dynamics by Elastic Strain-Gradient in Semiconductors. Adv. Mater. 2014, 26, 2572-2579.

41. Fu, X.; Jacopin, G.; Shahmohammadi, M.; Liu, R.; Benameur, M.; Ganière, J.-D.; Feng, J.; Guo, W.; Liao, Z.-M.; Deveaud, B.; et al. Exciton Drift in Semiconductors under Uniform Strain Gradients: Application to Bent ZnO Microwires. ACS Nano 2014, 4, 3412-3420.

42. Xue, H.; Pan, N.; Li, M.; Wu, Y.; Wang, X.; Hou, J. G. Probing the Strain Effect on Near Band Edge Emission of a Curved ZnO Nanowire via Spatially Resolved Cathodoluminescence. Nanotechnology 2010, 21, 215701.

43. Park, J. B.; Hong, W.-K.; Bae, T. S.; Sohn, J. I.; Cha, S. N.; Kim, J. M.; Yoon, H.; Lee, T. Strain Effects in a Single $\mathrm{ZnO}$ Microwire with Wavy Configurations. Nanotechnology 2013, 24, 455703 .

44. Reynolds, D. C.; Litton, C. W.; Collins, T. C. Optical Properties of ZnO. In Zinc Oxide Materials for Electronic and Optoelectronic Device Applications; Litton, C. W., Reynolds, D. C., Collins, T. C., Eds.; Wiley, 2011; p 51.

45. Gupta, V.; Mansingh, A. Breakdown Mechanism in Insulating Zinc Oxide Film. Proc. 11th IEEE Int. Symp. Appl. Ferroelectr. 1998, 113-116.

46. Pikus, G. E. Symmetry and Strain-Induced Effects in Semiconductors. Sov. Phys. Solid State 1964, 6, 261[translated in English in Fiz. Tverd. Tela 1964, 6, 324].

47. Bateman, T. B. Elastic Moduli of Single-Crystalline Zinc Oxide. J. Appl. Phys. 1962, 33, 3309-3312.

48. Muth, J. F.; Kolbas, R. M.; Sharma, A. K.; Oktyabrsky, S.; Narayan, J. Excitonic Structure and Absorption Coefficient Measurements of $\mathrm{ZnO}$ Single Crystal Epitaxial Films Deposited by Pulsed Laser Deposition. J. Appl. Phys. 1999, 85, 7884-7887.

49. Kanaya, K.; Okayama, S. Penetration and Energy-Loss Theory of Electrons in Solid Targets. J. Phys. D 1972, 5 , 43-58.

50. Watanabe, K.; Nakamura, Y.; Ichikawa, M. Conductive Optical-Fibre STM Probe for Local Excitation and Collection of Cathodoluminescence at Semiconductor Surfaces. Opt. Exp. 2013, 21, 19261-19268.

51. Watanabe, K.; Nakamura, Y.; Ichikawa, M. Spatial Resolution of Imaging Contaminations on the GaAs Surface by Scanning Tunneling Microscope-Cathodoluminescence Spectroscopy. Appl. Surf. Sci. 2008, 254, 7737-7741.

52. Nakamura, Y.; Amari, S.; Naruse, N.; Mera, Y.; Maeda, K.; Ichikawa, M. Self-Assembled Epitaxial Growth of High Density $\beta-\mathrm{FeSi}_{2}$ Nanodots on $\mathrm{Si}(001)$ and their Spatially Resolved Optical Absorption Properties. Cryst. Growth Des. 2008, 8, 3019-3023.

53. Watanabe, K.; Nokuo, T.; Chen, J.; Sekiguchi, T. Local Electrical Properties of n-AllnAs/i-GalnAs Electron Channel 
Structures Characterized by the Probe-Electron-BeamInduced Current Technique. Microscopy 2014, 63, 161166.

54. Liu, K. H.; Gao, P.; Xu, Z.; Bai, X. D.; Wang, E.-G. In-Situ Probing Electrical Response on Bending of ZnO Nanowires inside Transmission Electron Microscope. Appl. Phys. Lett. 2008, 92, 213105.

55. Nakamura, Y.; Murayama, A.; Ichikawa, M. Epitaxial Growth of High Quality Ge Films on Si (001) Substrates by Nanocontact Epitaxy. Cryst. Growth Des. 2011, 11, 3301-3305.

56. Minamisawa, R. A.; Süess, M. J.; Spolenak, R.; Faist, J.; David, C.; Gobrecht, J.; Bourdelle, K. K.; Sigg, H. Top-Down Fabricated Silicon Nanowires under Tensile Elastic Strain up to 4.5\%. Nat. Commun. 2012, 3, 1096.

57. Volk, J.; Nagata, T.; Erdérlyi, R.; Bársony, I.; Tóth, A. L.; Lukács, I. E.; Czigány, Z.; Tomimoto, H.; Shingaya, Y.; Chikyow, T. Highly Uniform Epitaxial ZnO Nanorods Arrays for Nanopiezotronics. Nanoscale Res. Lett. 2009, 4, 699-704.

58. Watanabe, K.; Oh, S.; Volk, J.; Nagata, T.; Wakayama, Y.; Sekiguchi, T. Multi-Directional Nano-Rods Growths and their Growth Mode Interactions Visualized and Modeled Using SEM-Cathodoluminescence Microscopy. Nat. Commun., to be submitted for publication. 